\title{
CREATION OPERATORS FOR THE FATEEV-ZAMOLODCHIKOV SPIN CHAIN
}

\author{
M. JIMBO, T. MIWA AND F. SMIRNOV \\ Dedicated to Ludwig Faddeev on the occasion of his eightieth birthday
}

\begin{abstract}
In our previous works on the XXZ chain of spin one half, we have studied the problem of constructing a basis of local operators whose members have simple vacuum expectation values. For this purpose a pair of fermionic creation operators have been introduced. In this article we extend this construction to the spin one case. We formulate the fusion procedure for the creation operators, and find a triplet of bosonic as well as two pairs of fermionic creation operators. We show that the resulting basis of local operators satisfies the dual reduced qKZ equation.
\end{abstract}

\section{INTRODUCTION}

In the series of papers [1, 2] Boos and Korepin put forward a conjecture that the correlation functions of an infinite anti-ferromagnetic XXX chain of spin $1 / 2$ can be expressed in terms of sums with rational coefficients of products of Riemann zeta function evaluated at odd integer points. This provides a considerable progress with respect to the multiple integral representations of [3, 4]. Later the conjecture was generalised to the XXZ chain. Considering the inhomogeneous case also proved to be very useful [5].

Proving the Boos-Korepin conjecture was the original motivation for the series of works of H. Boos, Y. Takeyama and the authors [6, 7, 8, 9]. In the final paper of this series [10] the conjecture was proved in a considerably generalised form. Namely, we have shown that the correlation function of the XXZ spin chain with the generalised Gibbs ensemble in the sense of [11] are expressed in terms of the Taylor coefficients of two functions, depending on one and two variables. These functions are defined by the thermodynamic characteristics of the generalised Gibbs ensemble alone. However, we emphasise that the construction involved in the process of the proof is, in our opinion, much more important than the conjecture itself. Let us explain this point.

First let us fix some terminology. Instead of dealing with the XXZ spin chain, it is more convenient for us to consider the six-vertex model equivalent to it. In the latter formulation, the object studied in [10] is the partition function on a cylinder carrying a certain defect, which we interpret as an insertion of a quasi-local operator. Assigning to each such insertion the corresponding partition function, we obtain a functional $Z$ defined on the space of quasi-local operators. Let us call the directrix of the cylinder the space direction, and the generatrix the Matsubara direction. In the space direction we considered only spin $1 / 2$, while in the Matsubara direction we allowed arbitrary spins and inhomogeneities. So, strictly speaking, it is not quite correct to call $Z$ the partition function of the six-vertex model, but we hope this should not be a problem for the reader. 
The main problem solved in [9] consists in providing a proper description of the space of quasi-local operators. It was shown that this space can be created by three kinds of one-parametric families of operators: a bosonic one, $t^{*}(\zeta)$, and two fermionic ones, $b^{*}(\zeta)$ and $c^{*}(\zeta)$. Since the bosonic operator in question is rather trivial, we call this the fermionic construction. We are mostly interested in quasi-local operators of spin 0 . They are obtained by acting with an equal number of $b^{*}$ s and $c^{*}$ 's and an arbitrary number of $t^{*}$ 's on the "primary field" $q^{2 \alpha S(0)}$ (this notation will be explained in Section [2). For example, the expression

$$
b^{*}\left(\zeta_{1}\right) c^{*}\left(\zeta_{2}\right)\left(q^{2 \alpha S(0)}\right)
$$

should be considered not as one quasi-local operator, but as a family of quasi-local operators of different lengths obtained by the Taylor expansion of (1.1) in $\zeta_{1}^{2}-1, \zeta_{2}^{2}-1$. On the other hand the value of our functional $Z$ on (1.1) is given by the function of two variables mentioned above, $\omega\left(\zeta_{1}, \zeta_{2}\right)$. The situation is reminiscent of CFT: operators of different lengths have a universal formula for the expectation values. This led us in the years 2008-2009 to the effort of relating the fermionic construction to CFT.

In the paper [12] we have shown, together with H. Boos, that in the case of the homogeneous spin 1/2 Matsubara chain the fermionic construction allows the scaling limit, providing the fermionic description of $c<1 \mathrm{CFT}$. The functional $Z$ gives the three-point functions. This has far-reaching consequences. Our construction can be generalised to the case of an inhomogeneous spin chain in the space direction. In the scaling limit, this gives the one point functions of the sine-Gordon model. Then we use the results obtained for CFT in order to normalise the operators, i.e. to identify them with the CFT primary fields and descendants. This step is necessary for the application of one point functions in the perturbed CFT, to study the short distance behaviour of multi-point correlators via OPE. All this is explained in detail in [13, 14]. Thus the fermionic construction allows us to solve the problem of computing the one point functions in the sine-Gordon model. The importance of this problem was pointed out by Al. Zamolodchikov [15. Further S. Negro and one of the authors showed [16, 17] that in the Liouville CFT the fermionic basis provides a neat solution to the reflection relations of [18]. This gives one more evidence in favour of the fermionic basis.

There is one more interesting circumstance which is worth mentioning. This is a general property of integrable models. The function $\omega\left(\zeta_{1}, \zeta_{2}\right)$ gives not only the value of the functional $Z$ on the family of quasi-local operators (1.1), but also the value of a similar functional on one operator for an inhomogeneous chain with only two space sites and the same Matsubara chain. This point has been discussed in [9, 10], but we decided to elucidate it once again in Section 2 in a more consistent way.

Now we come to the subject of the present paper. It is interesting to generalise our previous results to the integrable anisotropic spin 1 chain which was found by Fateev and Zamolodchikov [19] or, in our language, rather to the corresponding 19-vertex model. We avoid using the term "spin 1 XXZ model" because even being often used by specialists in integrable models, this term is misleading for general physics audience. The first thing to do in this direction is to prove an analogue of the Boos-Korepin conjecture, i.e., to show that the correlation functions on a finite number of sites do not really require multiple integrals, which become really boundless in this case [20]. This was essentially done by 
A. Klümper, D. Nawrah and J. Suzuki in the recent work [21]. Actually the construction of [10] already allows us to treat the spin 1 representation in the Matsubara direction. Hence the only thing to be done is to consider the fusion in the space direction. This requires proving that certain singularities cancel. In [21] this was done case by case. On the physicists' level of rigour, the cancellation must hold generally because the object under the consideration is well-defined. The paper [21] gives a solid evidence that the correlation functions in the infinite volume are expressed in terms of certain elementary functions, so the zeta function is not needed in this case.

However we are interested in the generalisation of our previous construction. Namely we want a construction of the space of quasi-local operators in terms of multi-parametric families similar to the one given by fermions. Our goals include the description of the super-symmetric CFT, the computation of one point functions in the super-symmetric sine Gordon model, and the solution of the reflection relations for the super-symmetric Liouville model. In the present paper we make a modest step in this direction: we show that on the lattice the space of quasi-local operators is created by one simple boson $\mathbf{t}^{*}(\zeta)$, four fermions $\mathbf{b}^{*}(\zeta), \overline{\mathbf{b}}^{*}(\zeta) \mathbf{c}^{*}(\zeta), \overline{\mathbf{c}}^{*}(\zeta)$, and three bosons $\mathbf{j}^{+}(\zeta), \mathbf{j}^{0}(\zeta), \mathbf{j}^{-}(\zeta)$. More exactly, similarly to the spin $1 / 2$ case, we have to take their Taylor expansions in $\zeta^{2}-1$.

Symbolically the spin 1 creation operators are defined in terms of the spin $1 / 2$ creation operators as follows:

$$
\begin{aligned}
& \mathbf{j}^{+}(\zeta)=b^{*}\left(\zeta q^{1 / 2}\right) b^{*}\left(\zeta q^{-1 / 2}\right), \\
& \mathbf{j}^{-}(\zeta)=-c^{*}\left(\zeta q^{1 / 2}\right) c^{*}\left(\zeta q^{-1 / 2}\right), \\
& \mathbf{j}^{0}(\zeta)=b^{*}\left(\zeta q^{1 / 2}\right) c^{*}\left(\zeta q^{-1 / 2}\right)+c^{*}\left(\zeta q^{1 / 2}\right) b^{*}\left(\zeta q^{-1 / 2}\right), \\
& \mathbf{b}^{*}(\zeta)=b^{*}\left(\zeta q^{1 / 2}\right) t^{*}\left(\zeta q^{-1 / 2}\right)+b^{*}\left(\zeta q^{-1 / 2}\right), \\
& \overline{\mathbf{b}}^{*}(\zeta)=b^{*}\left(\zeta q^{1 / 2}\right)+t^{*}\left(\zeta q^{1 / 2}\right) b^{*}\left(\zeta q^{-1 / 2}\right), \\
& \mathbf{c}^{*}(\zeta)=c^{*}\left(\zeta q^{1 / 2}\right) t^{*}\left(\zeta q^{-1 / 2}\right)+c^{*}\left(\zeta q^{-1 / 2}\right), \\
& \overline{\mathbf{c}}^{*}(\zeta)=c^{*}\left(\zeta q^{1 / 2}\right)+t^{*}\left(\zeta q^{1 / 2}\right) c^{*}\left(\zeta q^{-1 / 2}\right) .
\end{aligned}
$$

The precise sense of these formulas will be discussed in Section 3.2. In fact we first define the corresponding operators for an inhomogeneous chain where independent evaluation parameters are attached to each site, then use them to define creation operators for the homogeneous chain. Our main tool is the Russian doll construction, see the formulae (3.4), (3.5) below. There are two issues involved in the definition (1.2). First, we must ensure that the combinations (1.2) are well-defined. This concerns the case of $\mathbf{j}^{0}(\zeta)$, where each term has a singularity and only the sum makes sense. Second, the product of spin 1 creation operators on the inhomgeneous chain have singularities on the diagonal. This is a new feature which was absent in the spin $1 / 2$ case. It causes a problem in the definition of the creation operators for the homogeneous chain. We are forced to subtract the singular terms and replace the naïve product by a normal ordered product. There is a certain arbitrariness in the choice of functions used to define the subtraction. We fix the ambiguity by demanding that, in the infinite volume limit in the Matsubara direction, all quasi-local operators constructed by the creation operators have vanishing expectation values. This requirement is needed for the consistency with CFT as explained in [12. 
The expectation values are essentially given in terms of the function $\omega(\zeta, \xi)$. So the most natural way to satisfy the above requirement would be to compute the infinite Matsubara limit of this function. For that matter, some results from 21 may be useful. We leave the investigation of this function for another publication and proceed differently here. It is known that in the infinite volume limit both in the Matsubara and the space direction keeping a finite number of inhomogeneities in space, the density matrix is given by a solution to the reduced qKZ equation [3]. We show that our creation operators satisfy the dual reduced qKZ equation. This fact is interesting by itself, and it also allows us to fix the infinite Matsubara normalisation.

The text is organised as follows. In Section 2 we explain that, for quasi-local operators of a certain specific form, the computation of expectation values on an infinite homogeneous chain can be reduced to that on a finite inhomogeneous chain. In Section 3, after reviewing the creation operators for spin $1 / 2$, we introduce the fusion procedure to construct creation operators of spin 1 . In Section 4 we show that the resulting quasi-local operators satisfy the dual reduced qKZ equation. In Section 5 we give a brief summary. In Appendix A we review the definition and properties of the creation operators in the spin $1 / 2$ case. In Appendix B we discuss the regularity property of the products of creation operators.

Throughout this paper we fix $q=e^{\pi i \nu}$ where $0<\nu<1$.

\section{FUNCTIONAL ON QUASI-LOCAL OPERATORS IN A QUANTUM SPIN CHAIN: "INFINITE HOMOGENEOUS" FROM "FINITE INHOMOGENEOUS"}

We begin with a simple construction, which we shall explain for the vertex models related to the algebra $U_{q}\left(\widehat{\mathfrak{s l}}_{2}\right)$, but which is actually model independent. Our aim is to construct a family of quasi-local operators on an infinite homogeneous chain using an inductive limit of monodromy matrices. Then, we reduce the computation of certain functionals on this family to that of certain matrix elements of monodromy matrices on a finite inhomogeneous spin chain. Overall in this paper we shall use the normalised trace $\mathrm{Tr}$, so that for a $d$-dimensional space we have

$$
\operatorname{Tr}(x)=\frac{1}{d} \operatorname{tr}(x)
$$

$\operatorname{tr}(x)$ being the usual trace.

Consider a vertex model on a square lattice on an infinite cylinder. The sites along the generatrix (we call this the space direction) will be counted by the index $j$, while the sites along the directrix (called the Matsubara direction) are counted by the index $\mathbf{m}$. The total number of sites in the Matsubara direction is denoted by $\mathbf{n}$. Consider an exactly solvable model on this cylinder whose Boltzmann weights are given by $R$-matrices in the tensor product of two evaluation representations of $U_{q}\left(\widehat{\mathfrak{s l}}_{2}\right)$. We shall consider a model which is homogeneous in the space direction consisting of the $d$-dimensional representation with an equal evaluation parameter, say 1. In the Matsubara direction the representations may be of different dimensions $d_{\mathbf{m}}$ and may carry different evaluation parameters $\tau_{\mathbf{m}}$ (inhomogeneous model). In the following, we will replace the role of the infinite homogeneous spin chain in the space direction by an auxiliary finite inhomogeneos spin chain, while keeping the spin chain in the Matsubara direction. 
We consider the partition function on the cylinder with an insertion of a quasi-local operator in the space direction. Let us give the precise definition.

Consider a finite-type quantum algebra $U_{q}\left(\mathfrak{s l}_{2}\right)$ and its affinisation $U_{q}\left(\widehat{\mathfrak{s l}}_{2}\right)$. We define two spaces:

$$
\mathfrak{H}_{\mathrm{S}}=\bigotimes_{j=-\infty}^{\infty} \mathbb{C}^{d}, \quad \mathfrak{H}_{\mathbf{M}}=\bigotimes_{\mathbf{m}=1}^{\mathbf{n}} \mathbb{C}^{d_{\mathbf{m}}}
$$

The first definition poses a certain problem, but we shall avoid it working rather with operators acting on $\mathfrak{H}_{\mathbf{S}}$ than with this space itself. Among these operators there are well-defined quasi-local ones which we are going to describe. Denote by $H$ the Cartan generator of $U_{q}\left(\mathfrak{s l}_{2}\right)$, and take a complex number $\alpha$.

Denote by $H_{j}$ the Cartan generator acting on $V_{j}=\mathbb{C}^{d}$, the $j$-th copy of the tensor product in the space direction. We shall consider the 'primary operators':

$$
q^{\alpha H(0)}, \quad H(k)=\sum_{j=-\infty}^{k} H_{j}
$$

and their descendants:

$$
q^{\alpha H(0)} \mathcal{O}
$$

where the operator $\mathcal{O}$ acts non-trivially only on a finite number of sites. We call them quasi-local operators.

Introduce another complex number $\kappa$ which we shall couple with

$$
H(\infty)=\sum_{j=-\infty}^{\infty} H_{j}
$$

The partition function with an insertion of a quasi-local operator is defined as follows. Consider the monodromy matrix in the Matsubara direction

$$
T_{j, \mathbf{M}}(\zeta)=\prod_{\mathbf{m}=\mathbf{1}}^{\curvearrowleft} R_{j, \mathbf{m}}\left(\zeta / \tau_{\mathbf{m}}\right)=R_{j, \mathbf{n}}\left(\zeta / \tau_{\mathbf{n}}\right) \cdots R_{j, \mathbf{1}}\left(\zeta / \tau_{\mathbf{1}}\right),
$$

and the space-Matsubara monodromy matrix:

$$
T_{\mathbf{S}, \mathbf{M}}=\prod_{j=-\infty}^{\infty} T_{j, \mathbf{M}}(1)=\cdots T_{-1, \mathbf{M}}(1) T_{0, \mathbf{M}}(1) T_{1, \mathbf{M}}(1) \cdots
$$

Let $T_{\mathbf{M}}(\zeta, \kappa)=\operatorname{Tr}_{j}\left(T_{j, \mathbf{M}}(\zeta) q^{\kappa H_{j}}\right)$ denote the corresponding transfer matrix (recall that we use the normalised trace). The main object of our study is

$$
Z_{\mathbf{n}}^{\kappa}\left\{q^{\alpha H(0)} \mathcal{O}\right\}=\frac{\operatorname{Tr}_{\mathbf{M}} \operatorname{Tr}_{\mathbf{S}}\left(T_{\mathbf{S}, \mathbf{M}} q^{\kappa H(\infty)+\alpha H(0)} \mathcal{O}\right)}{\operatorname{Tr}_{\mathbf{M}} \operatorname{Tr}_{\mathbf{S}}\left(T_{\mathbf{S}, \mathbf{M}} q^{\kappa H(\infty)+\alpha H(0)}\right)}
$$

This defines a linear functional on the space of quasi-local operators. We assume without loss of generality that $[H(\infty), \mathcal{O}]=0$, since the numerator vanishes if $\mathcal{O}$ carries a non-zero spin, i.e., $[H(\infty), \mathcal{O}]=2 s \mathcal{O}$ where $s \neq 0$. The values of this functional depend not only on $\kappa$ and $\mathbf{n}$, but also on the dimensions of the spaces in the Matsubara direction and the 
corresponding inhomogeneities $\tau_{\mathbf{m}}$. We suppress this dependence in order to simplify the notation.

In the generic situation there are no infinities in $Z_{\mathbf{n}}^{\kappa}\left\{q^{\alpha H(0)} \mathcal{O}\right\}$. Indeed, suppose that the transfer matrix $T_{\mathbf{M}}(1, \kappa)$ has a unique eigenvector $|\kappa\rangle$ such that the corresponding eigenvalue $T(1, \kappa)$ has the maximal absolute value. Similarly let $\langle\kappa+\alpha|$ be an eigencovector of $T_{\mathbf{M}}(1, \kappa+\alpha)$ with the eigenvalue $T(1, \kappa+\alpha)$ possessing the same property. Impose the generality condition

$$
\langle\kappa+\alpha \mid \kappa\rangle \neq 0
$$

Suppose that

$$
q^{\alpha H(0)} \mathcal{O}=q^{\alpha H(j-1)} X_{[j, k]},
$$

where $X_{[j, k]}$ acts as identity outside some interval $[j, k](k \geq j)$. Then we say that $q^{\alpha H(0)} \mathcal{O}$ is supported on the interval $[j, k]$. We have

$$
Z_{\mathbf{n}}^{\kappa}\left\{q^{\alpha H(0)} \mathcal{O}\right\}=\frac{T(1, \kappa+\alpha)^{j-1}}{T(1, \kappa)^{k}} \cdot \frac{\left\langle\kappa+\alpha\left|\operatorname{Tr}_{[j, k]}\left(T_{[j, k], \mathbf{M}} q^{\kappa H_{[j, k]} X_{[j, k]}}\right)\right| \kappa\right\rangle}{\langle\kappa+\alpha \mid \kappa\rangle},
$$

where

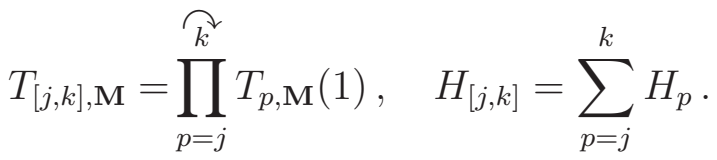

It is clear that by a simple redefinition of operators we can restrict our consideration to the case $j=1$, i.e. when $\mathcal{O}$ is supported on the interval $[1, k]$. We ask ourselves a question: is there a way for constructing such $\mathcal{O}$, which is useful for the evaluation of the functional (2.3) and which takes integrability into account?

Consider an additional evaluation representation $V_{c}$ of the same dimension as $V_{j}$ used in the space direction. Let us equip $V_{c}$ with the evaluation parameter $\zeta$. Introduce the adjoint $R$-matrix

$$
\mathbb{R}_{c, j}(\zeta)(\bullet)=R_{c, j}(\zeta) \bullet R_{c, j}(\zeta)^{-1}
$$

Define further the following, rather formal, object

$$
\mathbb{T}_{c,[1, \infty]}(\zeta)=\prod_{j=1}^{\infty} \mathbb{R}_{c, j}(\zeta)
$$

Consider a linear operator

$$
y_{c,[1, k]}: \operatorname{End}\left(V_{1} \otimes \cdots \otimes V_{k}\right) \longrightarrow \operatorname{End}\left(V_{1} \otimes \cdots \otimes V_{k} \otimes V_{c}\right) .
$$

Our goal is to make sense of the coefficients $y^{(j)}$ in the expansion

$$
\operatorname{Tr}_{c}\left(\mathbb{T}_{c,[1, \infty)}(\zeta) y_{c,[1, k]}\left(X_{[1, k]}\right)\right)=\sum_{j=0}^{\infty}\left(\zeta^{2}-1\right)^{j} y^{(j)}\left(X_{[1, k]}\right)
$$

as an operator sending $X_{[1, k]} \in \operatorname{End}\left(V_{1} \otimes \cdots \otimes V_{k}\right)$ to

$$
y^{(j)}\left(X_{[1, k]}\right) \in \operatorname{End}\left(V_{[1, \infty)}\right)=\lim _{l \rightarrow \infty} \operatorname{End}\left(V_{1} \otimes \cdots \otimes V_{l}\right) .
$$


Here the limit in the right hand side is the inductive limit with respect to the inclusion

$$
\operatorname{End}\left(V_{1} \otimes \cdots \otimes V_{l}\right) \otimes \mathrm{id} \subset \operatorname{End}\left(V_{1} \otimes \cdots \otimes V_{l+1}\right) .
$$

Remark. The above inductive limit $l \rightarrow \infty$ concerns the right end of the interval $[1, l]$. In [9] this was called the right reduction. As for the left reduction, i.e., $k \rightarrow-\infty$ for the interval $[k, m]$, there is nothing more to add than the argument given in 9 .

In what follows we shall consider functions of $\zeta$ as functions of $w=\zeta^{2}-1$ near $w=0$. For example, we understand $\zeta^{a}=(1+w)^{a / 2}$, the branch is such that this function equals 1 at $w=0$.

It will be convenient to use

$$
\check{\mathbb{R}}_{i, j}(\zeta)=\mathbb{R}_{i, j}(\zeta) \mathbb{P}_{i, j}
$$

where $\mathbb{P}_{i, j}$ stands for the adjoint action of the permutation.

We have

$$
\left.\check{\mathbb{R}}_{i, j}(\zeta)\right|_{\zeta^{2}=1}=I
$$

hence

$$
\check{\mathbb{R}}_{i, j}(\zeta)=I+\left(\zeta^{2}-1\right) \mathbf{r}_{i, j}(\zeta)
$$

where the operator $\mathbf{r}_{i, j}(\zeta)$ is regular at $\zeta^{2}=1$, and possesses the

\section{Property:}

$$
\mathbf{r}_{i, j}(\zeta)(X)=0
$$

if the operator $X$ acts trivially on the $i$-th and the $j$-th components of the tensor product.

Using this property one shows, following [9], that for any finite $l>k$

$$
\begin{aligned}
& \operatorname{Tr}_{c}\left(\mathbb{T}_{c,[1, l]}(\zeta) y_{c,[1, k]}\left(X_{[1, k]}\right)\right) \\
& =\sum_{j=k}^{l-1}\left(\zeta^{2}-1\right)^{j-k} \mathbf{r}_{j+1, j}(\zeta) \cdots \mathbf{r}_{k+2, k+1}(\zeta) \mathbb{T}_{k+1,[1, k]}(\zeta)\left(y_{k+1,[1, k]}\left(X_{[1, k]}\right)\right) \\
& +O\left(\left(\zeta^{2}-1\right)^{l-k}\right)
\end{aligned}
$$

as Taylor series in $\zeta^{2}-1$.

Let us emphasise the most important property of (2.6): there are no gaps in the product of successive matrices $\mathbf{r}_{j+1, j}(\zeta), \ldots, \mathbf{r}_{k+2, k+1}(\zeta)$. This is a consequence of the above Property.

So, the inductive limit $l \rightarrow \infty$ for these power series is well-defined: every Taylor coefficient stabilises for sufficiently large $l$, and we define

$$
\begin{aligned}
& \operatorname{Tr}_{c}\left(\mathbb{T}_{c,[1, \infty)}(\zeta) y_{c,[1, k]}\left(X_{[1, k]}\right)\right) \\
& =\sum_{j=k}^{\infty}\left(\zeta^{2}-1\right)^{j-k} \mathbf{r}_{j+1, j}(\zeta) \cdots \mathbf{r}_{k+2, k+1}(\zeta) \mathbb{T}_{k+1,[1, k]}(\zeta) y_{k+1,[1, k]}\left(X_{[1, k]}\right)
\end{aligned}
$$

Notice that we can allow $y_{k+1,[1, k]}$ to depend analytically on $\zeta$ provided there is no singularity at $\zeta^{2}=1$, i.e., if the Taylor expansion is possible. 
The above procedure can be iterated, and leads to the following construction. Consider auxiliary spaces $V_{c_{1}}, \cdots, V_{c_{k}}$ with the evaluation parameters $\zeta_{1}, \cdots, \zeta_{k}$. Consider further

$$
f_{c_{1}, \cdots, c_{k}} \in \operatorname{End}\left(V_{c_{1}} \otimes \cdots \otimes V_{c_{k}}\right) .
$$

Then we have a $k$-parametric generating function of local operators:

$$
\operatorname{Tr}_{c_{1}, \cdots, c_{k}}\left(\mathbb{T}_{c_{1},[1, \infty]}\left(\zeta_{1}\right) \cdots \mathbb{T}_{c_{k},[1, \infty]}\left(\zeta_{k}\right)\left(f_{c_{1}, \cdots, c_{k}}\right)\right)
$$

Remark. We can allow $f_{c_{1}, \cdots, c_{k}}$ to depend analytically on $\zeta_{j}$ provided they have no singularities at $\zeta_{j}^{2}=1$ and on the diagonals $\zeta_{i}^{2}=\zeta_{j}^{2}$, i.e., if the Taylor expansion is possible.

Following HGSIII, Lemma 3.1, one computes the functional $Z_{\mathbf{n}}^{\kappa}$ on the local operators given by these generating functions:

$$
\begin{aligned}
& Z_{\mathbf{n}}^{\kappa}\left\{q^{\alpha H(0)} \operatorname{Tr}_{c_{1}, \cdots, c_{k}}\left(\mathbb{T}_{c_{1},[1, \infty]}\left(\zeta_{1}\right) \cdots \mathbb{T}_{c_{k},[1, \infty]}\left(\zeta_{k}\right)\left(f_{c_{1}, \cdots, c_{k}}\right)\right)\right\} \\
& =\frac{\left\langle\kappa+\alpha\left|\operatorname{Tr}_{c_{1}, \cdots, c_{k}}\left(T_{c_{1}, \mathbf{M}}\left(\zeta_{1}, \kappa\right) \cdots T_{c_{k}, \mathbf{M}}\left(\zeta_{k}, \kappa\right) f_{c_{1}, \cdots, c_{k}}\right)\right| \kappa\right\rangle}{\langle\kappa+\alpha \mid \kappa\rangle \prod_{p=1}^{k} T\left(\zeta_{p}, \kappa\right)},
\end{aligned}
$$

where both left and right hand sides are understood as Taylor series in $\zeta_{j}^{2}-1$.

So, the values of the functionals $Z_{\mathbf{n}}^{\kappa}$ on the generating function (2.8) are expressed through the matrix elements of the products of matrices $T_{c_{j}, \mathrm{M}}\left(\zeta_{j}, \kappa\right)$. In other words the computation on the infinite homogeneous chain $\otimes_{j=1}^{\infty} \mathbb{C}^{d}$ is reduced to that on the finite inhomogeneous chain $\otimes_{j=1}^{k} \mathbb{C}^{d}$ with the inhomogeneities $\zeta_{1}, \cdots, \zeta_{k}$. This is a nice observation, but it becomes useful only if we know a really good way to compute these matrix elements. Up to now this was known only in the case where we have two-dimensional representations in the space direction. In the next section we shall repeat briefly this construction, and generalise it in order to include the case of three-dimensional representations.

\section{BASIS OF QUASI-LOCAL OPERATORS FOR SPIN 1 CHAIN}

3.1. The case of spin $\mathbf{1} / \mathbf{2}$. In the case of spin $1 / 2$, a family of 'good' basis $\left\{f_{c_{1}, \cdots, c_{k}}\right\}$ of finite inhomogeneous chains was found in [9]. In this section we briefly outline this construction. We shall deal with the space of quasi-local operators slightly more general than in the previous section,

$$
\mathcal{W}^{(\alpha)}=\bigoplus_{s \in \mathbb{Z}} \mathcal{W}_{\alpha-s, s}
$$

where $\mathcal{W}_{\alpha-s, s}$ consists of quasi-local operators $q^{(\alpha-s) H(0)} \mathcal{O}$ such that $\mathcal{O}$ has spin $s$. We shall also need the Cauchy-type kernel

$$
\psi(\zeta, \alpha)=\zeta^{\alpha} \frac{\zeta^{2}+1}{2\left(\zeta^{2}-1\right)}
$$

and its 'primitive' function $\Delta_{\zeta}^{-1} \psi(\zeta, \alpha)$ with respect to the $q$-difference operator

$$
\Delta_{\zeta} f(\zeta)=f(q \zeta)-f\left(q^{-1} \zeta\right)
$$


We fix possible arbitrariness in quasi-constants defining for $0<\operatorname{Re}(\alpha)<1 / \nu$

$$
\Delta_{\zeta}^{-1} \psi(\zeta, \alpha)=-\frac{1}{8 i} V P \int_{-\infty}^{\infty} \zeta^{i k+\alpha} \frac{\operatorname{coth} \frac{\pi k}{2}}{\sinh \pi \nu(k-i \alpha)} d k,
$$

and then continuing analytically with respect to $\alpha$.

Consider the two-dimensional auxiliary spaces $V_{c_{j}}\left(\zeta_{j}\right)$ with evaluation parameters $\zeta_{j}$, $j=1,2, \cdots$. Our construction is based on the following linear maps introduced in [9]

$$
g_{c_{k}}^{\epsilon}\left(\zeta_{k}\right): \operatorname{End}\left(V_{c_{1}}\left(\zeta_{1}\right) \otimes \cdots \otimes V_{c_{k-1}}\left(\zeta_{k-1}\right)\right) \rightarrow \operatorname{End}\left(V_{c_{1}}\left(\zeta_{1}\right) \otimes \cdots \otimes V_{c_{k}}\left(\zeta_{k}\right)\right),
$$

where $\epsilon_{k} \in\{ \pm, 0\}$. In Appendix $\AA$ we summarise the definition of the operators $g_{c}^{\epsilon}(\zeta)$ and their properties. The operator $g_{c_{k}}^{\epsilon_{k}}\left(\zeta_{k}\right)$ depends also on the other parameters $\epsilon_{j}$ and $\zeta_{j}$, $1 \leq j \leq k-1$. For brevity we suppress this dependence from the notation. Sometimes we write the operators in a different order. For example

$$
g_{c_{1}}^{\epsilon_{1}}\left(\zeta_{1}\right) g_{c_{2}}^{\epsilon_{2}}\left(\zeta_{2}\right)(I) \in \operatorname{End}\left(V_{c_{2}}\left(\zeta_{2}\right) \otimes V_{c_{1}}\left(\zeta_{1}\right)\right)
$$

means that we first act with an operator which 'adds' the space $V_{c_{2}}\left(\zeta_{2}\right)$, and then 'add' $V_{c_{1}}\left(\zeta_{1}\right)$ further. The following commutation relations hold, see (A.24) and the discussions therein:

$$
g_{c_{1}}^{\epsilon_{1}}\left(\zeta_{1}\right) g_{c_{2}}^{\epsilon_{2}}\left(\zeta_{2}\right)=(-)^{\epsilon_{1} \epsilon_{2}} \mathbb{R}_{c_{1}, c_{2}}\left(\zeta_{1} / \zeta_{2}\right) g_{c_{2}}^{\epsilon_{2}}\left(\zeta_{2}\right) g_{c_{1}}^{\epsilon_{1}}\left(\zeta_{1}\right) .
$$

Remark. The operators $g_{c}^{\epsilon}(\zeta)$ used here are slightly different from those of [9]. In Appendix A.2 we denote the latter by $g_{\mathrm{rat}, c}^{\epsilon}(\zeta)$. The coefficients of the operators $g_{\mathrm{rat}, c}^{\epsilon}(\zeta)$ are rational functions in the evaluation parameters (up to an overall power). In contrast, those of $g_{c}^{\epsilon}(\zeta)$ involve the transcendental function (3.2) (see e.g. Example 2 in Section A.1). However it is $g_{c}^{\epsilon}(\zeta)$ which has a better behaviour in relation to CFT [12], and in this paper we use it exclusively.

Following [9] introduce operators $x^{\epsilon *}(\zeta)$ acting on $\mathcal{W}^{(\alpha)}$ by

$$
x^{\epsilon *}(\zeta)\left(q^{(\alpha-s) H(0)} \mathcal{O}\right)=q^{(\alpha-s-\epsilon) H(0)} \operatorname{Tr}_{c}\left(\mathbb{T}_{c,[n+1, \infty)}(\zeta) g_{c}^{\epsilon}(\zeta)\left(\mathcal{O}_{[1, n]}\right)\right),
$$

where $\mathcal{O}$ is supported on $[1, n]$ and has spin $s$. Usually we write

$$
b^{*}(\zeta)=x^{+*}(\zeta), \quad c^{*}(\zeta)=x^{-*}(\zeta), \quad t^{*}(\zeta)=x^{0 *}(\zeta) .
$$

In [9], [10] we used boldface letters, but we shall reserve them for the spin 1 case in the present paper. One concludes that the operators $x^{\epsilon *}(\zeta)$ are well defined on $\mathcal{W}^{(\alpha)}$ setting $\mathbb{T}_{c,[1, k]}(\zeta) y_{c,[1, k]}=g_{c}^{\epsilon}(\zeta)$ in (2.7).

We have the commutation relations

$$
x^{\epsilon_{1 *}}\left(\zeta_{1}\right) x^{\epsilon_{2} *}\left(\zeta_{2}\right)=(-)^{\epsilon_{1} \epsilon_{2}} x^{\epsilon_{2} *}\left(\zeta_{2}\right) x^{\epsilon_{1 *}}\left(\zeta_{1}\right) .
$$

We construct operators on the inhomogeneous chains by applying the Russian doll principle, i.e., recursively in the form

$$
g_{c_{k}}^{\epsilon_{k}}\left(\zeta_{k}\right) \cdots g_{c_{1}}^{\epsilon_{1}}\left(\zeta_{1}\right)(I) .
$$

It is known that the expression (3.4) is regular at $\zeta_{j}^{2}=1$ and on the diagonal $\zeta_{j}^{2}=\zeta_{k}^{2}$, $j \neq k$ (see [9], Lemma 3.8). 
The consecutive application of the operators $x^{\epsilon *}(\zeta)$ yields (set $n=0$ in (A.22) )

$$
\begin{aligned}
& x^{\epsilon_{k} *}\left(\zeta_{k}\right) \cdots x^{\epsilon_{1} *}\left(\zeta_{1}\right)\left(q^{\alpha H(0)}\right) \\
& =\operatorname{Tr}_{c_{1}, \cdots, c_{k}}\left(\mathbb{T}_{c_{1},[1, \infty)}\left(\zeta_{1}\right) \cdots \mathbb{T}_{c_{k},[1, \infty)}\left(\zeta_{k}\right) g_{c_{k}}^{\epsilon_{k}}\left(\zeta_{k}\right) \cdots g_{c_{1}}^{\epsilon_{1}}\left(\zeta_{1}\right)(I)\right) q^{\left(\alpha-\sum \epsilon_{p}\right) H(0)} .
\end{aligned}
$$

We call this "dressed Russian doll" formula because it allows to define the action of operators on End $\left(\mathfrak{H}_{\mathrm{S}}\right)$ by covering the Russian doll in the auxiliary spaces End $\left(V_{c_{1}} \otimes \cdots \otimes V_{c_{k}}\right)$ with the adjoint monodromy matrices and tracing out the auxiliary spaces.

The virtue of (3.5) is that their expectation values can be described explicitly. The main formula of [10], (1.12), can be written as follows

$$
\begin{aligned}
& \frac{\left\langle\kappa+\alpha\left|\operatorname{Tr}_{c_{1}, \cdots, c_{k}}\left(T_{c_{1}, \mathbf{M}}\left(\zeta_{1}\right) \cdots T_{c_{k}, \mathbf{M}}\left(\zeta_{k}\right) g_{c_{k}}^{\epsilon_{k}}\left(\zeta_{k}\right) \cdots g_{c_{1}}^{\epsilon_{1}}\left(\zeta_{1}\right)(I)\right)\right| \kappa\right\rangle}{\prod_{p=1}^{k} T\left(\zeta_{p}, \kappa\right)\langle\kappa+\alpha \mid \kappa\rangle} \\
& =\prod_{j: \epsilon_{j}=0} \rho\left(\zeta_{j}\right) \times\left.\operatorname{det}\left(\omega\left(\zeta_{r}, \zeta_{s}\right)\right)\right|_{\substack{r: \epsilon_{r}=+s: \epsilon_{s}=-}} .
\end{aligned}
$$

Here $\rho(\zeta)$ is a ratio of the left and right eigenvalues of the Matsubara transfer-matrix,

$$
\rho(\zeta)=\frac{T(\zeta, \kappa+\alpha)}{T(\zeta, \kappa)},
$$

and the function $\omega(\zeta, \xi)$ is given in Appendix A. Here we mention only the properties most essential for our goals.

The function $\omega(\zeta, \xi)$ consists of two pieces,

$$
\omega(\zeta, \xi)=\omega_{\text {reg }}(\zeta, \xi)+\omega_{\text {trans }}(\zeta, \xi)
$$

The first piece $(\xi / \zeta)^{\alpha} \omega_{\text {reg }}(\zeta, \xi)$ is a meromorphic function of $\zeta^{2}$ and $\xi^{2}$ with simple poles located at the zeros of $T(\zeta, \kappa) T(\xi, \kappa)$ and is regular elsewhere. The second piece is given by

$$
\begin{aligned}
& \omega_{\text {trans }}(\zeta, \xi)=\frac{1}{4} \frac{1}{T(\zeta, \kappa) T(\xi, \kappa)}\left(a(\xi) d(\zeta) \psi(q \zeta / \xi, \alpha)-a(\zeta) d(\xi) \psi\left(q^{-1} \zeta / \xi, \alpha\right)\right) \\
& +(1+\rho(\zeta) \rho(\xi)) \Delta_{\zeta}^{-1} \psi(\zeta / \xi, \alpha)-\rho(\zeta) \Delta_{\zeta}^{-1} \psi\left(q^{-1} \zeta / \xi, \alpha\right)-\rho(\xi) \Delta_{\zeta}^{-1} \psi(q \zeta / \xi, \alpha) .
\end{aligned}
$$

It has simple poles at $\zeta / \xi=q^{ \pm 1}$, with the residue

$$
\begin{aligned}
& \operatorname{res}_{\zeta^{\prime}=\zeta} \omega\left(q^{ \pm 1 / 2} \zeta^{\prime}, q^{\mp 1 / 2} \zeta\right) \frac{d \zeta^{\prime 2}}{\zeta^{\prime 2}}= \pm \mathcal{N}(\zeta) \cdot \frac{1}{2}\left(1+\frac{T^{(1)}(\zeta, \kappa+\alpha)}{T^{(1)}(\zeta, \kappa)}\right), \\
& \mathcal{N}(\zeta)=\frac{3}{4} \frac{T^{(1)}(\zeta, \kappa)}{T\left(q^{-1 / 2} \zeta, \kappa\right) T\left(q^{1 / 2} \zeta, \kappa\right)} .
\end{aligned}
$$

The appearance of fused spin 1 transfer matrices $T^{(1)}(\zeta, \kappa), T^{(1)}(\zeta, \kappa+\alpha)$ in this formula is very important. In deriving (3.8) we used (3.7),

$$
\Delta^{-1} \psi(\zeta, \alpha)= \pm \frac{1}{2} \psi\left(q^{\mp 1} \zeta, \alpha\right)+O(1) \quad\left(\zeta \rightarrow q^{ \pm 1}\right)
$$

and the fusion relations for the transfer matrix eigenvalues

$$
3 T^{(1)}(\zeta, \kappa)=4 T\left(\zeta q^{1 / 2}, \kappa\right) T\left(\zeta q^{-1 / 2}, \kappa\right)-a\left(\zeta q^{1 / 2}\right) d\left(\zeta q^{-1 / 2}\right) .
$$

(Recall again that the transfer matrices are defined via the normalised trace). 
3.2. Fusion of spin $\mathbf{1 / 2}$ operators. Certainly, the road from spin $1 / 2$ to spin 1 passes through fusion. In this subsection we consider the inhomogeneous chain of spin 1.

Let us start from some generalities. The spin 1 representation is realised as a $U_{q}\left(\widehat{\mathfrak{s l}}_{2}\right)$ submodule

$$
V_{\{1,2\}^{+}}(\zeta) \subset V_{1}\left(\zeta^{-}\right) \otimes V_{2}\left(\zeta^{+}\right)
$$

where $\zeta^{ \pm}=q^{ \pm 1 / 2} \zeta$. For definiteness we normalise the $R$ matrix in such a way that

$$
R_{1,2}(q)=D_{1,2} \mathcal{P}_{1,2}^{+}, \quad \operatorname{res}_{\zeta=q^{-1}} R_{1,2}(\zeta) \frac{d \zeta^{2}}{\zeta^{2}}=\mathcal{P}_{1,2}^{-}
$$

where $\mathcal{P}_{1,2}^{ \pm}=\left(\mathcal{P}_{1,2}^{ \pm}\right)^{2}$ are the projectors onto the symmetric and anti-symmetric subspaces, and $D_{1,2}$ is a diagonal matrix which commutes with $\mathcal{P}_{1,2}^{+}$.

Consider an operator $f_{1,2} \in \operatorname{End}\left(V_{1}\left(\zeta^{-}\right) \otimes V_{2}\left(\zeta^{+}\right)\right)$. If its image is contained in the subspace $V_{\{1,2\}}+(\zeta)$, then by restriction we obtain

$$
f_{\{1,2\}^{+}}=\left.f_{1,2}\right|_{V_{\{1,2\}^{+}}(\zeta)} \in \text { End } V_{\{1,2\}^{+}}(\zeta) .
$$

It means that End $V_{\{1,2\}}+(\zeta)$ is a subquotient

$$
\text { End } V_{\{1,2\}^{+}}(\zeta) \simeq \mathcal{P}_{1,2}^{+} \operatorname{End}\left(V_{1}\left(\zeta^{-}\right) \otimes V_{2}\left(\zeta^{+}\right)\right) \bmod \quad \mathcal{P}_{1,2}^{+} \operatorname{End}\left(V_{1}\left(\zeta^{-}\right) \otimes V_{2}\left(\zeta^{+}\right)\right) \mathcal{P}_{1,2}^{-} .
$$

More generally, let $f_{c_{1}, \cdots, c_{2 n}}$ be an operator on $V_{c_{1}}\left(\zeta_{1}^{-}\right) \otimes V_{c_{2}}\left(\zeta_{1}^{+}\right) \otimes \cdots \otimes V_{c_{2 n-1}}\left(\zeta_{n}^{-}\right) \otimes V_{c_{2 n}}\left(\zeta_{n}^{+}\right)$, $\zeta_{j}^{ \pm}=q^{ \pm 1 / 2} \zeta_{j}$, and suppose that

$$
f_{c_{1}, \cdots, c_{2 n}}=\mathcal{P}_{2 j-1,2 j}^{+} f_{c_{1}, \cdots, c_{2 n}} \quad(j=1, \cdots, n) .
$$

Let $f_{\mathbf{c}_{1}, \cdots, \mathbf{c}_{\mathbf{n}}}$ be its restriction to $V_{\mathbf{c}_{1}} \otimes \cdots \otimes V_{\mathbf{c}_{\mathbf{n}}}$ where we write $\mathbf{c}_{j}=\left\{c_{2 j-1}, c_{2 j}\right\}^{+}$for brevity. From the fusion relation

$$
R_{1, *}\left(q^{-1 / 2} \zeta\right) R_{2, *}\left(q^{1 / 2} \zeta\right) \mathcal{P}_{1,2}^{+}=R_{\{1,2\}^{+}, *}(\zeta)
$$

we obtain the following relation between the expectation values

$$
\begin{aligned}
& \frac{\left\langle\kappa+\alpha\left|\operatorname{Tr}_{c_{1}, \cdots, c_{2 n}}\left(T_{c_{1}, \mathbf{M}}\left(\zeta_{1}^{-}, \kappa\right) T_{c_{2}, \mathbf{M}}\left(\zeta_{1}^{+}, \kappa\right) \cdots T_{c_{2 n-1}, \mathbf{M}}\left(\zeta_{n}^{-}, \kappa\right) T_{c_{2 n}, \mathbf{M}}\left(\zeta_{n}^{+}, \kappa\right) f_{c_{1}, \cdots, c_{2 n}}\right)\right| \kappa\right\rangle}{\langle\kappa+\alpha \mid \kappa\rangle \prod_{p=1}^{n} T\left(\zeta_{p}^{-}, \kappa\right) T\left(\zeta_{p}^{+}, \kappa\right)} \\
& =\prod_{j=1}^{n} \mathcal{N}\left(\zeta_{j}\right) \times \frac{\left\langle\kappa+\alpha\left|\operatorname{Tr}_{\mathbf{c}_{1}, \cdots, \mathbf{c}_{n}}\left(T_{\mathbf{c}_{1}, \mathbf{M}}\left(\zeta_{1}, \kappa\right) \cdots T_{\mathbf{c}_{n}, \mathbf{M}}\left(\zeta_{n}, \kappa\right) f_{\mathbf{c}_{1}, \cdots, \mathbf{c}_{n}}\right)\right| \kappa\right\rangle}{\langle\kappa+\alpha \mid \kappa\rangle \prod_{p=1}^{n} T^{(1)}\left(\zeta_{p}, \kappa\right)},
\end{aligned}
$$

where $\mathcal{N}(\zeta)$ is given by (3.9).

Now we discuss the fusion of operators $g_{c}^{\epsilon}(\zeta)$. Consider some linear combination $\mathbf{g}_{c_{2}, c_{1}}^{\delta}(\zeta)$ of $g_{c_{2}}^{\epsilon_{2}}\left(\zeta q^{1 / 2}\right) g_{c_{1}}^{\epsilon_{1}}\left(\zeta q^{-1 / 2}\right)$ where $\epsilon_{1}+\epsilon_{2}$ is fixed. In addition to (A.5-7), it is convenient to use the operator $g_{c}^{\overline{0}}(\zeta)$ meaning

$$
g_{c}^{\overline{0}}(\zeta)(X)=X \otimes \mathrm{id}_{c}
$$

for which the value of $\epsilon$ is understood to be 0 . 
We say $\mathbf{g}_{c_{2}, c_{1}}^{\delta}(\zeta)$ is admissible if

$$
\mathbf{g}_{c_{2}, c_{1}}^{\delta}(\zeta)(I)=\mathcal{P}_{c_{1}, c_{2}}^{+} \mathbf{g}_{c_{2}, c_{1}}^{\delta}(\zeta)(I) .
$$

For admissible operators the following holds:

$$
\mathbf{g}_{c_{2 n}, c_{2 n-1}}^{\delta_{k}}\left(\zeta_{n}\right) \cdots \mathbf{g}_{c_{2}, c_{1}}^{\delta_{1}}\left(\zeta_{1}\right)(I)=\mathcal{P}_{c_{2 k-1}, c_{2 n}}^{+} \cdots \mathcal{P}_{c_{1}, c_{2}}^{+} \mathbf{g}_{c_{2 n}, c_{2 n-1}}^{\delta_{n}}\left(\zeta_{k}\right) \cdots \mathbf{g}_{c_{2}, c_{1}}^{\delta_{1}}\left(\zeta_{1}\right)(I) .
$$

To see this take $X \in \operatorname{End}\left(V_{[1, n]}\right)$ as in Subsection A.1, and note that the $R$ matrix symmetry (A.17) implies

$$
\begin{aligned}
& g_{c}^{\epsilon}\left(\zeta_{1} \mid \xi_{1}, \cdots, \xi_{j}, q \xi_{j}, \cdots, \xi_{n}\right)\left(\mathcal{P}_{j+1, j}^{+} X\right) \\
& =\mathcal{P}_{j+1, j}^{+} g_{c}^{\epsilon}\left(\zeta_{1} \mid \xi_{1}, \cdots, \xi_{j}, q \xi_{j}, \cdots, \xi_{n}\right)\left(\mathcal{P}_{j+1, j}^{+} X\right)
\end{aligned}
$$

provided the specialisation $\left.g_{c}^{\epsilon}\left(\zeta_{1} \mid \xi_{1}, \cdots, \xi_{n}\right)\right|_{\xi_{j+1}=q \xi_{j}}$ is well defined. Using the commutation relations (3.3) we can bring any $\mathbf{g}_{c_{2 j}, c_{2 j-1}}^{\delta_{j}}\left(\zeta_{k}\right)$ to the right and use (3.14), then the symmetriser goes to the left because of (3.16).

We choose the index $\delta$ to take values in $(i, j), i, j=1,2,3$. There are two admissible operators $\mathbf{g}_{c_{2}, c_{1}}^{\delta}(\zeta)$ which we can construct immediately:

$$
\mathbf{g}_{c_{2}, c_{1}}^{(3,1)}(\zeta)=g_{c_{2}}^{+}\left(\zeta q^{1 / 2}\right) g_{c_{1}}^{+}\left(\zeta q^{-1 / 2}\right), \quad \mathbf{g}_{c_{2}, c_{1}}^{(1,3)}(\zeta)=g_{c_{2}}^{-}\left(\zeta q^{1 / 2}\right) g_{c_{1}}^{-}\left(\zeta q^{-1 / 2}\right) .
$$

Indeed, $g_{c_{2}}^{ \pm}\left(\zeta q^{1 / 2}\right) g_{c_{1}}^{ \pm}\left(\zeta q^{-1 / 2}\right)(I)$ are proportional to $\sigma_{c_{2}}^{ \pm} \sigma_{c_{1}}^{ \pm}$, which obviously satisfy (3.14). These two operators look as a part of a triplet, so we want to find one more operator of similar type which does not change the total spin. Certainly, it should be built of $g_{c_{2}}^{+} g_{c_{1}}^{-}$ and $g_{c_{2}}^{-} g_{c_{1}}^{+}$. Taking care of singularities we come with the following proposal:

$$
\mathbf{g}_{c_{2}, c_{1}}^{(2,2)}(\zeta)=\lim _{\substack{\xi_{1} \rightarrow \zeta q^{-1 / 2} \\ \xi_{2} \rightarrow \zeta q^{1 / 2}}}\left(\left(q \xi_{1} / \xi_{2}\right)^{\alpha} g_{c_{2}}^{+}\left(\xi_{2}\right) g_{c_{1}}^{-}\left(\xi_{1}\right)+\left(q \xi_{1} / \xi_{2}\right)^{-\alpha} g_{c_{2}}^{-}\left(\xi_{2}\right) g_{c_{1}}^{+}\left(\xi_{1}\right)\right) .
$$

In Appendix B we show that the limit (3.17) is well-defined. By a direct computation using (A.10), (A.11) we find

$$
\begin{aligned}
& \mathbf{g}_{c_{2}, c_{1}}^{(2,2)}(\zeta)(I) \\
& =-\left(q^{\alpha}-q^{-\alpha}\right) \mathcal{P}_{1,2}^{+}\left(\frac{q+q^{-1}}{2\left(q-q^{-1}\right)}\left(q^{\alpha} \tau_{1}^{+} \tau_{2}^{+}+q^{-\alpha} \tau_{1}^{-} \tau_{2}^{-}\right)+\frac{q^{\alpha}+q^{-\alpha}}{q^{2}-q^{-2}}\left(\tau_{1}^{+} \tau_{2}^{-}+\tau_{1}^{-} \tau_{2}^{+}\right)\right) .
\end{aligned}
$$

Hence it is admissible.

Now we pass to the fermions. It is easy to see that the following combinations are admissible:

$$
\begin{aligned}
& \mathbf{g}_{c_{2}, c_{1}}^{(1,2)}(\zeta)=g_{c_{2}}^{-}\left(\zeta q^{1 / 2}\right) g_{c_{1}}^{0}\left(\zeta q^{-1 / 2}\right)+g_{c_{1}}^{-}\left(\zeta q^{-1 / 2}\right) \\
& \mathbf{g}_{c_{2}, c_{1}}^{(2,1)}(\zeta)=g_{c_{2}}^{+}\left(\zeta q^{1 / 2}\right) g_{c_{1}}^{0}\left(\zeta q^{-1 / 2}\right)+g_{c_{1}}^{+}\left(\zeta q^{-1 / 2}\right) \\
& \mathbf{g}_{c_{2}, c_{1}}^{(2,3)}(\zeta)=g_{c_{2}}^{0}\left(\zeta q^{1 / 2}\right) g_{c_{1}}^{-}\left(\zeta q^{-1 / 2}\right)+g_{c_{2}}^{-}\left(\zeta q^{1 / 2}\right) \\
& \mathbf{g}_{c_{2}, c_{1}}^{(3,2)}(\zeta)=g_{c_{2}}^{0}\left(\zeta q^{1 / 2}\right) g_{c_{1}}^{+}\left(\zeta q^{-1 / 2}\right)+g_{c_{2}}^{+}\left(\zeta q^{1 / 2}\right)
\end{aligned}
$$

We add two more simple operators. The unit operator for the fused chain

$$
\mathbf{g}_{c_{2}, c_{1}}^{(1,1)}(\zeta)=\mathrm{id},
$$


and

$$
\mathbf{g}_{c_{2}, c_{1}}^{(3,3)}(\zeta)=g_{c_{2}}^{0}\left(\zeta q^{1 / 2}\right) g_{c_{1}}^{0}\left(\zeta q^{-1 / 2}\right)
$$

After restricting to the spin 1 subspaces we obtain

$$
\begin{aligned}
\mathbf{g}_{\mathbf{c}_{k}}^{\delta}(\zeta): \operatorname{End} & \left(V_{\mathbf{c}_{1}}\left(\zeta_{1}\right) \otimes \cdots \otimes V_{\mathbf{c}_{k-1}}\left(\zeta_{k-1}\right)\right) \\
& \longrightarrow \operatorname{End}\left(V_{\mathbf{c}_{1}}\left(\zeta_{1}\right) \otimes \cdots \otimes V_{\mathbf{c}_{k-1}}\left(\zeta_{k-1}\right) \otimes V_{\mathbf{c}_{k}}(\zeta)\right)
\end{aligned}
$$

where $\mathbf{c}_{j}=\left\{c_{2 j-1}, c_{2 j}\right\}^{+}$.

3.3. Creation operators for spin 1. From now on, for economy of symbols we use indices $c_{j}$ to label the three-dimensional spaces. There should not be a confusion with the two-dimensional case because for the operators we use letters of different style.

It is easy to see that $\mathbf{g}_{c}^{(i, j)}(\zeta)(I)$, with $i, j=1,2,3$, provide nine independent operators on one site. Using this fact it should not be hard to prove the completeness for the inhomogeneous case in the spirit of [22].

Following our previous logic we would like now to define operators on an infinite chain of spin 1 from $\mathbf{g}_{c}^{(i, j)}(\zeta)$. A naïve attempt would be to use the formula

$$
\begin{aligned}
& \mathbf{x}^{\left(i_{k}, j_{k}\right)}\left(\zeta_{k}\right) \cdots \mathbf{x}^{\left(i_{1}, j_{1}\right)}\left(\zeta_{1}\right)\left(q^{\alpha H(0)}\right) \\
& =\operatorname{Tr}_{c_{1}, \cdots, c_{k}}\left[\mathbb{T}_{c_{1},[1, \infty)}\left(\zeta_{1}\right) \cdots \mathbb{T}_{c_{k},[1, \infty)}\left(\zeta_{k}\right) \mathbf{g}_{c_{k}}^{\left(i_{k}, j_{k}\right)}\left(\zeta_{k}\right) \cdots \mathbf{g}_{c_{1}}^{\left(i_{1}, j_{1}\right)}\left(\zeta_{1}\right)(I)\right] \cdot q^{\left(\alpha-\sum_{p}\left(j_{p}-i_{p}\right)\right) H(0)} .
\end{aligned}
$$

However, this does not work directly: the right hand side does not admit a Taylor expansion because of singularities on the diagonals. Let us explain this.

Consider for example the case

$$
\mathbf{g}_{\{3,4\}^{+}}^{(3,1)}\left(\zeta_{2}\right) \mathbf{g}_{\{1,2\}^{+}}^{(1,3)}\left(\zeta_{1}\right)\left(q^{\alpha H(0)}\right)=\lim g_{4}^{+}\left(\xi_{4}\right) g_{3}^{+}\left(\xi_{3}\right) g_{2}^{-}\left(\xi_{2}\right) g_{1}^{-}\left(\xi_{1}\right)(I),
$$

where we set

$$
\xi_{2 j}=q^{1 / 2} \zeta_{j}^{\prime}, \quad \xi_{2 j-1}=q^{-1 / 2} \zeta_{j}, \quad(j=1,2)
$$

and take the limit $\zeta_{j}^{\prime} \rightarrow \zeta_{j}$. In order to unveil the singularity it is easier to deal with the expectation values than with the operators themselves. Take a Matsubara chain with arbitrary parameters $\boldsymbol{\tau}_{\mathbf{m}}$ and $d_{\mathbf{m}}$ and compute the expectation value according to (3.6)

$$
\frac{\left\langle\kappa+\alpha\left|g_{4}^{+}\left(\xi_{4}\right) g_{3}^{+}\left(\xi_{3}\right) g_{2}^{-}\left(\xi_{2}\right) g_{1}^{-}\left(\xi_{1}\right)(I)\right| \kappa\right\rangle}{\langle\kappa+\alpha \mid \kappa\rangle}=\omega\left(\xi_{4}, \xi_{1}\right) \omega\left(\xi_{3}, \xi_{2}\right)-\omega\left(\xi_{4}, \xi_{2}\right) \omega\left(\xi_{3}, \xi_{1}\right) \text {. }
$$

In the above limit, only the first term develops singularities. Let us set

$$
\bar{f}(\zeta, \xi)=\frac{1}{2}\left(1+\frac{T^{(1)}(\xi, \kappa+\alpha)}{T^{(1)}(\xi, \kappa)}\right) \psi(\zeta / \xi, \alpha)
$$

and write

$$
\begin{aligned}
\omega\left(q^{1 / 2} \zeta, q^{-1 / 2} \xi\right) & =\mathcal{N}(\xi) \bar{f}(\zeta, \xi)+\omega^{\prime \prime}(\zeta, \xi), \\
\omega\left(q^{-1 / 2} \zeta, q^{1 / 2} \xi\right) & =\mathcal{N}(\zeta) \bar{f}(\xi, \zeta)+\omega^{\prime}(\zeta, \xi),
\end{aligned}
$$


where $\omega^{\prime \prime}(\zeta, \xi), \omega^{\prime}(\zeta, \xi)$ are regular as $\zeta \rightarrow \xi$. From (3.8) we find

$$
\begin{aligned}
& \omega\left(\xi_{4}, \xi_{1}\right) \omega\left(\xi_{3}, \xi_{2}\right)=\mathcal{N}\left(\zeta_{1}\right) \mathcal{N}\left(\zeta_{2}\right) \bar{f}\left(\zeta_{2}, \zeta_{1}\right) \bar{f}\left(\zeta_{1}, \zeta_{2}\right) \\
& +\mathcal{N}\left(\zeta_{2}\right) \bar{f}\left(\zeta_{2}, \zeta_{1}\right)\left(-\omega^{\prime \prime}\left(\zeta_{2}, \zeta_{1}\right)+\omega^{\prime}\left(\zeta_{2}, \zeta_{1}\right)\right)+O(1)
\end{aligned}
$$

On the other hand, the expectation value of $\mathbf{g}^{(2,2)}(\zeta)$ reads

$$
\lim _{\substack{\xi_{2} \rightarrow q^{1 / 2} \zeta \\ \xi_{1} \rightarrow q^{-1 / 2} \zeta}}\left(\left(q \xi_{1} / \xi_{2}\right)^{\alpha} \omega\left(\xi_{2}, \xi_{1}\right)-\left(q \xi_{1} / \xi_{2}\right)^{-\alpha} \omega\left(\xi_{1}, \xi_{2}\right)\right)=\omega^{\prime \prime}(\zeta, \zeta)-\omega^{\prime}(\zeta, \zeta) .
$$

Now let us pass to the limit (3.20). The expectation values of the spin 1 chain is related to those of spin $1 / 2$ chain through (3.12). Comparing these, we find that the expectation value of

$$
\left(\mathbf{g}_{\{3,4\}^{+}}^{(3,1)}\left(\zeta_{2}\right) \mathbf{g}_{\{1,2\}^{+}}^{(1,3)}\left(\zeta_{1}\right)+\bar{f}\left(\zeta_{2}, \zeta_{1}\right) \bar{f}\left(\zeta_{1}, \zeta_{2}\right)-\bar{f}\left(\zeta_{2}, \zeta_{1}\right) \mathbf{g}^{(2,2)}\left(\zeta_{1}\right)\right)(I)
$$

is regular at $\zeta_{2} \rightarrow \zeta_{1}$.

Let us return to the operators $\mathbf{x}^{(i, j)}$. Normally we use different letters to denote them,

$$
\left(\mathbf{x}^{(i, j)}(\zeta)\right)_{i, j=1,2,3}=\left(\begin{array}{ccc}
\mathrm{id}, & \mathbf{c}^{*}(\zeta), & \mathbf{j}^{-}(\zeta) \\
\mathbf{b}^{*}(\zeta), & \mathbf{j}^{0}(\zeta), & \overline{\mathbf{c}}^{*}(\zeta) \\
\mathbf{j}^{+}(\zeta), & \mathbf{b}^{*}(\zeta), & \mathbf{t}^{*}(\zeta)
\end{array}\right)
$$

The formula (3.19) does not define $\mathbf{x}^{(i, j)}$ as operators acting on $\mathcal{W}^{(\alpha)}$ because of the singularities. However, the above investigation of singularities suggests the following construction.

Choose and fix a function $f(\zeta, \xi)$ which is regular in the vicinity of $\zeta / \xi \in \mathbb{R}_{>0}$ except for a simple pole with the residue

$$
\operatorname{res}_{\zeta=\xi} f(\zeta, \xi)=\frac{1}{2}\left(1+\mathbf{t}^{*}(\xi)\right) .
$$

Let us emphasise that $\mathbf{t}^{*}(\xi)$ is in the centre of the algebra of creation operators, hence we can manipulate it as a constant. Since in the above considerations the Matsubara chain was arbitrary, we conclude that as an operator we have (compare [23] where a similar argument is used)

$$
\mathbf{j}^{+}(\zeta) \mathbf{j}^{-}(\xi)=f(\zeta, \xi) \mathbf{j}^{0}(\xi)-f(\zeta, \xi) f(\xi, \zeta)+O(1) .
$$

Notice that changing the choice of $f(\zeta, \xi)$ does not affect this equation because $f(\zeta, \xi) f(\xi, \zeta)$ does not have a contribution to the simple pole term.

In the general case we have the following construction. Consider the bosonic operators, which obey the OPE for the current algebra $\widehat{\mathfrak{s l}}_{2}$ with the central charge 1 :

$$
\begin{aligned}
& \mathbf{j}^{+}(\zeta) \mathbf{j}^{-}(\xi)=f(\zeta, \xi) \mathbf{j}^{0}(\xi)-f(\zeta, \xi) f(\xi, \zeta) \\
& \mathbf{j}^{0}(\zeta) \mathbf{j}^{0}(\xi)=-2 f(\zeta, \xi) f(\xi, \zeta), \quad \mathbf{j}^{0}(\zeta) \mathbf{j}^{ \pm}(\xi)= \pm f(\zeta, \xi) \mathbf{j}^{ \pm}(\xi) .
\end{aligned}
$$

The appearance of this algebra is as mysterious as the appearance of fermions in the spin $1 / 2$ case. Consider further two pairs of fermions $\left(\mathbf{b}^{*}, \mathbf{c}^{*}\right),\left(\overline{\mathbf{b}}^{*}, \overline{\mathbf{c}}^{*}\right)$ which transform as 
$\mathfrak{s l}_{2}$-doublets, the non-trivial OPE being

$$
\begin{aligned}
& \mathbf{j}^{+}(\zeta) \mathbf{c}^{*}(\xi)=-f(\zeta, \xi) \mathbf{b}^{*}(\xi), \quad \mathbf{j}^{-}(\zeta) \mathbf{b}^{*}(\xi)=-f(\zeta, \xi) \mathbf{c}^{*}(\xi), \\
& \mathbf{j}^{0}(\zeta) \mathbf{b}^{*}(\xi)=f(\zeta, \xi) \mathbf{b}^{*}(\xi), \quad \mathbf{j}^{0}(\zeta) \mathbf{c}^{*}(\xi)=-f(\zeta, \xi) \mathbf{c}^{*}(\xi),
\end{aligned}
$$

and similarly for $\left(\overline{\mathbf{b}}^{*}, \overline{\mathbf{c}}^{*}\right)$. Finally, require the OPE between the two pairs of fermions:

$$
\mathbf{b}^{*}(\zeta) \overline{\mathbf{c}}^{*}(\xi)=-\left(1-\mathbf{t}^{*}(\xi)\right) f(\zeta, \xi), \quad \overline{\mathbf{b}}^{*}(\zeta) \mathbf{c}^{*}(\xi)=\left(1-\mathbf{t}^{*}(\xi)\right) f(\zeta, \xi) .
$$

The rest of the pairs do not develop singularities in the OPE. Using these OPE define the normal ordering : $\bullet$ : in the standard way. Then the normal ordered products of our operators define families of quasi-local operators by Taylor expansion in $\zeta^{2}-1$. For example,

$$
: \mathbf{j}^{+}(\zeta) \mathbf{j}^{-}(\xi):=\mathbf{j}^{+}(\zeta) \mathbf{j}^{-}(\xi)-f(\zeta, \xi) \mathbf{j}^{0}(\xi)+f(\zeta, \xi) f(\xi, \zeta)
$$

Another example is

$$
: \mathbf{j}^{0}(\zeta) \mathbf{b}^{*}(\xi) \overline{\mathbf{c}}^{*}(\eta):=\left\{\mathbf{j}^{0}(\zeta)-f(\zeta, \xi)+f(\zeta, \eta)\right\}: \mathbf{b}^{*}(\xi) \overline{\mathbf{c}}^{*}(\eta):
$$

Let us consider the Taylor coefficient in the normal ordered expressions as operators $\mathbf{x}_{k}^{(i, j)}$. Their commutation relations are derived as usual from the OPE.

Let us stress that we have tautologically defined the action of $\mathbf{x}_{k}^{(i, j)}$ on the Fock space created by themselves. We have little doubt that they span the entire space of quasi-local operators, but the proof of this statement is an unfinished task at this writing.

What is the best possible choice for the function $f(\zeta, \xi)$ ? Our final goal is the scaling limit which allows us to make contact with CFT. Namely, in the spin 1 case we expect to find in the limit a superconformal model with $c=3 / 2-12 \nu^{2} /(1-2 \nu)$ (in the spin $1 / 2$ case, this was simply a conformal model with $\left.c=1-6 \nu^{2} /(1-\nu)\right)$. This must be possible if one takes for Matsubara the homogeneous chain of three-dimensional spaces with parameters $\tau_{\mathrm{m}}$ all equal to $q^{1 / 2}$. Like in the spin-1/2 case [12] the operators $\mathbf{x}^{\delta}(\zeta)$ are supposed to produce descendants of the primary field obtained by the scaling limit of $q^{\alpha H(0)}$. The one point functions of descendants must vanish when the radius of the cylinder becomes infinite, i.e., when we get CFT on the plane. As explained in [12], for the spin-1/2 case similar property is satisfied even for the lattice model if we create quasi-local operators by $b^{*}(\zeta), c^{*}(\zeta), t^{*}(\zeta)-1$. This means that the expectation values of these quasi-local operators vanish for the infinite homogeneous Matsubara case. We wish to retain a similar property in the spin 1 case.

Namely, we want to fix the function $f(\zeta, \xi)$ in such a way that, in the limit when the homogeneous Matsubara chain becomes infinite, the expectation values vanish for the descendants created by normal ordered products of $\mathbf{x}^{\delta}(\zeta)$. One way of fixing this is to compute $\omega(\zeta, \xi)$, to take the limit of infinite homogeneous Matsubara case, and to see what happens. We would like to leave all such computations for another paper, but we shall comment later on the results from the related paper [21]. We shall take another way, 
which is interesting by itself, to fix the normalisation of operators in the infinite homogeneous chain. It is known [3] that the density matrix for the infinite homogeneous Matsubara case is given by special solutions to the reduced quantum Knizhnik-Zamolodchikov (rqKZ) equations. We shall check the quasi-local operators created by $\mathbf{x}^{\delta}(\zeta)$ against this description of the density matrix in the next section.

\section{REDUCED QKZ EQUATIONS}

Let us consider in general a model on a lattice which is infinite in both directions, whose rows and columns correspond to the same $d$-dimensional evaluation representation $V^{(d)}$ of $U_{q}\left(\widehat{\mathfrak{s l}}_{2}\right)$. The rqKZ equation is a general way of calculating correlation functions in this situation [3]. Assume that the evaluation parameters are $\tau_{\mathbf{m}}=q^{1 / 2}$ for all rows and 1 for all columns, except for $n$ consecutive columns to which independent parameters $\zeta_{1}, \cdots, \zeta_{n}$ are attached. Insert a local operator $X_{[1, n]}$ at these lines, and put a semi-infinite tail $q^{\alpha H(0)}$ (see [3] for more explication and graphical representation). The density matrix $h\left(\zeta_{1}, \zeta_{2}, \cdots, \zeta_{n}\right)$ is a linear functional which associates to $X_{[1, n]}$ the partition function

$$
h\left(\zeta_{1}, \zeta_{2}, \cdots, \zeta_{n}\right)\left(X_{[1, n]}\right)=\lim \frac{\left\langle\kappa+\alpha\left|\operatorname{Tr}_{1, \cdots, n}\left(T_{1, \mathbf{M}}\left(\zeta_{1}\right) \cdots T_{n, \mathbf{M}}\left(\zeta_{n}\right) X_{[1, n]}\right)\right| \kappa\right\rangle}{\prod_{p=1}^{n} T\left(\zeta_{p}, \kappa\right)\langle\kappa+\alpha \mid \kappa\rangle},
$$

where lim stays for the limit of infinite homogeneous Matsubara chain. It satisfies the rqKZ equation

$$
h\left(\zeta_{1}, \cdots, \zeta_{j} q^{-1}, \cdots \zeta_{n}\right)=h\left(\zeta_{1}, \zeta_{2}, \cdots, \zeta_{n}\right) \circ \mathbb{A}_{j,[1, n]}\left(\zeta_{1}, \cdots, \zeta_{n}\right),
$$

where $\mathbb{A}_{j,[1, n]}\left(\zeta_{1}, \cdots, \zeta_{n}\right)$ is defined as follows.

$$
\begin{aligned}
& \mathbb{A}_{j,[1, n]}\left(\zeta_{1}, \cdots, \zeta_{n}\right)\left(X_{[1, n]}\right) \\
& \quad=T_{j,[1, j-1]} q^{\alpha H_{j}} \theta_{j}\left(\theta_{j}\left(T_{j,[j+1, n]}^{-1}\right) X_{[1, n]} \theta_{j}\left(T_{j,[1, j-1]}^{-1}\right)\right) T_{j,[j+1, n]}, \\
& T_{j,[k, m]}=R_{j, m}\left(\zeta_{j} / \zeta_{m}\right) \cdots R_{j, k}\left(\zeta_{j} / \zeta_{k}\right) .
\end{aligned}
$$

Here $\theta(X)=C \cdot{ }^{t} X \cdot C^{-1}$, with $C: V^{(d) *}(q \zeta) \rightarrow V^{(d)}(\zeta)$ being an isomorphism of $U_{q}\left(\widehat{\mathfrak{s l}}_{2}\right)$ modules. For practical calculations it is convenient to rewrite (4.2) introducing two auxiliary spaces $V_{a}^{(d)}\left(\zeta_{j}\right)$ and $V_{b}^{(d)}\left(\zeta_{j}\right)$ :

$$
\begin{aligned}
& \mathbb{A}_{j,[1, n]}\left(\zeta_{1}, \cdots, \zeta_{n}\right)\left(X_{[1, n]}\right) \\
& =T_{j,[1, j-1]} q^{\alpha H_{j}} \operatorname{tr}_{a, b}\left(P_{b, j} T_{a,[j+1, n]}^{-1} \theta_{j}\left(X_{[1, n]}\right) T_{b,[1, j-1]}^{-1} P_{a, j}\right) T_{j,[j+1, n]}
\end{aligned}
$$

Let us mention some general properties of (4.2):

$$
\begin{aligned}
& \mathbb{A}_{n-1,[1, n-1]}\left(\zeta_{1}, \cdots, \zeta_{n-1}\right)\left(X_{[1, n-1]}\right)=\mathbb{A}_{n-1,[1, n]}\left(\zeta_{1}, \cdots, \zeta_{n}\right)\left(X_{[1, n-1]}\right), \\
& \mathbb{A}_{n,[1, n]}\left(\zeta_{1}, \cdots, \zeta_{n}\right)\left(X_{[1, n-1]}\right)=\mathbb{T}_{n,[1, n-1]}\left(\zeta_{n}\right)\left(q^{\alpha H_{n}} X_{[1, n-1]}\right), \\
& \mathbb{A}_{n,[1, n]}\left(\zeta_{1}, \cdots, \zeta_{n}\right)\left(\mathbb{T}_{n,[1, n-1]}\left(\zeta_{n} q^{-1}\right)\left(q^{\alpha H_{n}} X_{[1, n-1]}\right)\right)=X_{[1, n-1]} .
\end{aligned}
$$

In addition to (4.1), the density matrix has the following characteristic properties. 
1. In the limit $\zeta_{n}^{ \pm 1} \rightarrow \infty, h\left(\zeta_{1}, \zeta_{2}, \cdots, \zeta_{n}\right)$ reduces to $h\left(\zeta_{1}, \zeta_{2}, \cdots, \zeta_{n-1}\right)$ :

$$
\begin{aligned}
& h\left(\zeta_{1}, \zeta_{2}, \cdots, \zeta_{n}\right)\left(X_{[1, n-1]} \otimes x_{n}\right) \\
& \quad \rightarrow h\left(\zeta_{1}, \zeta_{2}, \cdots, \zeta_{n-1}\right)\left(X_{[1, n-1]}\right) \frac{\operatorname{Tr}_{n}\left(q^{-\alpha H_{n} / 2} x_{n}\right)}{\operatorname{Tr}_{n}\left(q^{-\alpha H_{n} / 2}\right)} .
\end{aligned}
$$

2. $h\left(\zeta_{1}, \zeta_{2}, \cdots, \zeta_{n}\right)$ is regular on the diagonals $\zeta_{i}=\zeta_{j}, i \neq j$.

Let us return to the model with spin $1 / 2$. In this case we have a distinguished basis of End $\left(V\left(\xi_{1}\right) \otimes \cdots \otimes V\left(\xi_{n}\right)\right)$,

$$
g^{\epsilon_{n}, \cdots, \epsilon_{1}}\left(\xi_{n}, \cdots, \xi_{1}\right)=g_{n}^{\epsilon_{n}}\left(\xi_{n}\right) \cdots g_{1}^{\epsilon_{1}}\left(\xi_{1}\right)(I) \quad\left(\epsilon_{1}, \cdots, \epsilon_{n} \in\{0, \overline{0},+,-\}\right)
$$

which satisfies the dual rqKZ equation (A.14) (recall the definition (3.13), and see Subsection A.3 for more explanations).

The roles of the rqKZ and the dual rqKZ equations are quite different. The rqKZ equation considered here is closely related to the qKZ equation 'of level -4 '. We are interested in one particular solution which describes the density matrix. In contrast, the dual rqKZ equation is closely related to the $\mathrm{qKZ}$ equation 'of level 0 '. It describes a good basis of the space of operators, in much the same way as in the original form factor equations [24].

Consider the pairing

$$
U^{\epsilon_{1}, \cdots, \epsilon_{n}}\left(\xi_{1}, \cdots, \xi_{n}\right)=h\left(\xi_{1}, \cdots, \xi_{n}\right)\left(g^{\epsilon_{n}, \cdots, \epsilon_{1}}\left(\xi_{n}, \cdots, \xi_{1}\right)\right),
$$

where $\epsilon_{j}= \pm, 0, \overline{0}$. The equations (4.1), (A.14) result in the following simple difference equation:

$$
U^{\epsilon_{1}, \cdots, \epsilon_{j}, \cdots, \epsilon_{n}}\left(\xi_{1} \cdots, \xi_{j} q^{-1}, \cdots, \xi_{n}\right)=(-1)^{\epsilon_{j}} U^{\epsilon_{1}, \cdots, \bar{\epsilon}_{j}, \cdots, \epsilon_{n}}\left(\xi_{1} \cdots, \xi_{j}, \cdots, \xi_{n}\right) .
$$

Suppose that $\xi_{1}, \cdots \xi_{j-1}, \xi_{j+1}, \cdots \xi_{n}$ are real. Then $U^{\epsilon_{1}, \cdots, \epsilon_{j}, \cdots, \epsilon_{n}}\left(\xi_{1} \cdots, \xi_{j}, \cdots, \xi_{n}\right)$ is a regular function of $\xi_{j}^{1 / \nu}$ since there are no singularities for $\arg \left(\xi_{j}\right) \leq \pi \nu / 2$. This function is odd if $\epsilon_{j}= \pm$, while for $\epsilon_{j}=0, \overline{0}$ it may contain even and odd parts. Further it does not vanish identically only if $\sum \epsilon_{p}=0$. Consider for definiteness the case $0<\alpha<2$. Suppose $\epsilon_{j}=+$. Then it can be shown that

$$
U^{\epsilon_{1}, \cdots, \epsilon_{n}}\left(\xi_{1}, \cdots, \xi_{n}\right)=\left\{\begin{array}{lll}
O(1) & \text { for } & \xi_{j} \rightarrow \infty \\
o(1) & \text { for } & \xi_{j} \rightarrow 0
\end{array}\right.
$$

The example (A.10) is instructive, actually it reflects well the generic situation. On the other hand it is easy to see that for $\epsilon_{j}=0, \overline{0}$

$$
U^{\epsilon_{1}, \cdots, \epsilon_{n}}\left(\xi_{1}, \cdots, \xi_{n}\right) \rightarrow U^{\epsilon_{1}, \cdots, \widehat{\epsilon}_{j}, \cdots, \epsilon_{n}}\left(\xi_{1}, \cdots, \widehat{\xi}_{j}, \cdots, \xi_{n}\right), \quad \text { for } \xi_{j} \rightarrow 0, \infty .
$$

From (4.9) and (4.10) we conclude that $U^{\epsilon_{1}, \cdots, \epsilon_{n}}\left(\xi_{1}, \cdots, \xi_{n}\right)$ vanishes if at least one of $\epsilon_{j}$ is + , and equals to 1 otherwise, i.e. when all $\epsilon_{j}$ equal 0 or $\overline{0}$. This means that for the infinite homogeneous spin 1/2 Matsubara spin chain we have

$$
\rho_{\infty}(\zeta)=1, \quad \omega_{\infty}(\zeta, \xi)=0 .
$$

This is an important property of the creation operators $b^{*}(\zeta), c^{*}(\zeta), t^{*}(\zeta)$. 
Now we want to test the fusion. In order to avoid confusions we put a superscript $1 / 2$ or 1 to the operator $\mathbb{A}_{j,[1, n]}$ for spin $1 / 2$ or of spin 1 . Using (A.15) and (A.16) we obtain

$$
\begin{aligned}
& g_{2 n}^{\epsilon}\left(\xi_{2 n}\right) g_{2 n-1}^{\epsilon^{\prime}}\left(\xi_{2 n-1}\right)\left(X_{[1,2 n-2]}\right) \\
& =g_{2 n}^{\epsilon}\left(\xi_{2 n}\right) \mathbb{A}_{2 n-1,[1,2 n-1]}^{(1 / 2)} g_{2 n-1}^{\bar{\epsilon}^{\prime}}\left(\xi_{2 n-1} q^{-1}\right)\left(X_{[1,2 n-2]}\right) \\
& =\mathbb{A}_{2 n-1,[1,2 n]}^{(1 / 2)}\left[g_{2 n,[1,2 n-1]}^{\epsilon}\left(\xi_{2 n}\right)\right]_{\xi_{2 n-1} \rightarrow \xi_{2 n-1} q^{-1}} g_{2 n-1}^{\bar{\epsilon}^{\prime}}\left(\xi_{2 n-1} q^{-1}\right)\left(X_{[1,2 n-2]}\right) \\
& =\mathbb{A}_{2 n-1,[1,2 n]}^{(1 / 2)}\left[\mathbb{A}_{2 n,[1,2 n]}^{(1 / 2)}\right]_{\xi_{2 n-1} \rightarrow \xi_{2 n-1} q^{-1}} \\
& \times\left[g_{2 n,[1,2 n-1]}^{\bar{\epsilon}}\left(\xi_{2 n}\right) g_{2 n-1}^{\bar{\epsilon}^{\prime}}\left(\xi_{2 n-1}\right)\left(X_{[1,2 n-2]}\right)\right]_{\xi_{2 n-1} \rightarrow \xi_{2 n-1} q^{-1}, \xi_{2 n} \rightarrow \xi_{2 n} q^{-1}}
\end{aligned}
$$

It is convenient to present the result of computation of the product of operators $\mathbb{A}$ introducing two auxiliary spaces,

$$
\begin{aligned}
& \mathbb{A}_{2 n-1,[1,2 n]}^{(1 / 2)}\left[\mathbb{A}_{2 n,[1,2 n]}^{(1 / 2)}\right]_{\xi_{2 n-1} \rightarrow \xi_{2 n-1} q^{-1}}\left(Y_{[1,2 n]}\right) \\
& =T_{2 n-1,[1,2 n-2]} T_{2 n,[1,2 n-2]} q^{\alpha\left(\sigma_{2 n-1}^{3}+\sigma_{2 n}^{3}\right)} \\
& \times \operatorname{tr}_{a, b}\left(P_{b, 2 n} P_{a, 2 n-1} R_{2 n, 2 n-1}\left(\xi_{2 n} / \xi_{2 n-1}\right) \theta_{2 n-1} \theta_{2 n}\left(Y_{[1,2 n]}\right) T_{b,[1,2 n-2]}^{-1} T_{a,[1,2 n-2]}^{-1}\right) R_{2 n-1,2 n}\left(\xi_{2 n-1} / \xi_{2 n}\right) .
\end{aligned}
$$

Consider now the limit $\xi_{2 n}=\zeta_{n} q^{1 / 2+\varepsilon}, \xi_{2 n-1}=\zeta_{n} q^{-1 / 2}$ with $\varepsilon \rightarrow 0$. Obviously $R_{2 n-1,2 n}\left(\xi_{2 n-1} / \xi_{2 n}\right)$ poses a problem because it contains a singularity. Suppose, however, that in this limit

$$
Y_{[1,2 n]}=\mathcal{P}_{2 n-1,2 n}^{+} Y_{[1,2 n]}+Z_{[1,2 n]} \cdot \varepsilon+o(\varepsilon),
$$

with some $Z_{[1,2 n]}$. Then

$$
\begin{aligned}
& \theta_{2 n-1} \theta_{2 n}\left(Y_{[1,2 n]}\right) R_{2 n-1,2 n}\left(\xi_{2 n-1} / \xi_{2 n}\right) \\
& =\theta_{2 n-1} \theta_{2 n}\left(Y_{[1,2 n]}\right) \mathcal{P}_{2 n-1,2 n}^{+}+\theta_{2 n-1} \theta_{2 n}\left(Z_{[1,2 n]}\right) \mathcal{P}_{2 n-1,2 n}^{-}+o(1) .
\end{aligned}
$$

Now the limit exists, we replace $R_{2 n, 2 n-1}\left(\xi_{2 n} / \xi_{2 n-1}\right)$ by $R_{2 n, 2 n-1}(q)$, the latter contains $\mathcal{P}_{2 n-1,2 n}^{+}$which starts to travel giving finally

$$
\begin{aligned}
& \mathbb{A}_{2 n-1,[1,2 n]}^{(1 / 2)}\left[\mathbb{A}_{2 n,[1,2 n]}^{(1 / 2)}\right]_{\xi_{2 n-1} \rightarrow \xi_{2 n-1} q^{-1}}\left(Y_{[1,2 n]}\right) \\
& \rightarrow \mathcal{P}_{2 n-1,2 n}^{+}\left(\mathbb{A}_{\{2 n-1,2 n\}^{+},[1,2 n-2]}^{(1)}\left(Y_{[1,2 n]}\right) \mathcal{P}_{2 n-1,2 n}^{+}+\mathbb{A}_{\{2 n-1,2 n\}^{+},[1,2 n-2]}^{(1)}\left(Z_{[1,2 n]}\right) \mathcal{P}_{2 n-1,2 n}^{-}\right) .
\end{aligned}
$$

Now recall that our combinations $\mathbf{g}_{2 n, 2 n-1}^{\delta}(\zeta)$ are exactly such that

$$
Y_{[1,2 n]}=\mathbf{g}_{2 n, 2 n-1}^{\delta}\left(\zeta_{n} q^{-1}\right)\left(X_{[1,2 n-2]}\right)
$$

satisfies the condition (4.12). Moreover, in view of (3.11) the last term in (4.13) does not count. So, we conclude that

$$
\mathbf{g}_{n, n-1}^{\delta}(\zeta)\left(X_{[1, n-1]}\right)=(-1)^{\delta} \mathbb{A}_{n,[1, n]}^{(1)} \mathbf{g}_{n, n-1}^{\bar{\delta}}\left(\zeta q^{-1}\right)\left(X_{[1, n-1]}\right),
$$

here and later we set

$$
(-1)^{(i, j)}=(-1)^{i+j}, \quad \overline{(i, j)}=(4-j, 4-i) .
$$


Now we proceed in the same way as for the spin-1/2 case, namely, we couple the solution to rqKZ equation with the operator created by $\mathbf{g}^{\delta_{j}}\left(\zeta_{j}\right)$ (the index $j$ refers to three-dimensional space now):

$$
U^{\delta_{1}, \cdots, \delta_{n}}\left(\zeta_{1}, \cdots, \zeta_{n}\right)=h\left(\zeta_{1}, \cdots, \zeta_{n}\right)\left(\mathbf{g}_{n}^{\delta_{n}}\left(\zeta_{n}\right) \cdots \mathbf{g}_{1}^{\delta_{1}}\left(\zeta_{1}\right)(I)\right) .
$$

As before this function satisfies the equation

$$
U^{\delta_{1}, \cdots \delta_{j}, \cdots, \delta_{n}}\left(\zeta_{1}, \cdots, \zeta_{j} q^{-1}, \cdots \zeta_{n}\right)=(-1)^{\delta_{j}} U^{\delta_{1}, \cdots \overline{\delta_{j}}, \cdots \delta_{n}}\left(\zeta_{1}, \cdots, \zeta_{j}, \cdots \zeta_{n}\right) .
$$

However, contrary to the spin-1/2 case we cannot conclude immediately that (4.15) is either 0 or 1 , since the operator created by $\mathbf{g}_{j}^{\delta}\left(\zeta_{j}\right)$ contains singularities on the diagonals. Following the discussion at the end of the previous section we want to eliminate them by normal ordering. But we do not want to spoil the difference relation. This will be achieved if we set

$$
f(\zeta, \xi)=\left(1+\mathbf{t}^{*}(\xi)\right) \frac{2(\zeta \xi)^{\frac{1}{\nu}}}{\nu\left(\zeta^{\frac{2}{\nu}}-\xi^{\frac{2}{\nu}}\right)}
$$

Notice that we put a function anti-periodic under $\zeta \rightarrow \zeta q$ in order to preserve the signs for fermions and bosons in (4.16). Plugging in (4.15) the normal ordered product of $\mathbf{g}^{\delta_{j}}\left(\zeta_{j}\right)$ we obtain 0 if some $\delta_{j}$ differ from $(1,1),(3,3)$, and 1 otherwise.

\section{Summary And Discussions}

We have shown how to construct the operators $\mathbf{b}^{*}(\zeta), \mathbf{c}^{*}(\zeta), \overline{\mathbf{b}}^{*}(\zeta), \overline{\mathbf{c}}^{*}(\zeta), \mathbf{j}^{ \pm}(\zeta), \mathbf{j}^{0}(\zeta)$ and $\mathbf{t}^{*}(\zeta)$ which create the quasi-local operators for the Fateev-Zamolodchikov spin chain. The linear functional $Z_{\mathbf{n}}^{\kappa}$ can be evaluated on these quasi-local fields using the formulae (1.2) from Introduction, but certain adjustment is needed. Namely, from the main text it is clear that we have to multiply formally the right hand side of $(1.2)$ by $\mathcal{N}(\zeta)^{-1}$ in order to change the normalisation of $Z_{\mathbf{n}}^{\kappa}$ to the one appropriate for the spin 1 case. Let us clarify this giving one example of the final formulae:

$$
Z_{\mathbf{n}}^{\kappa}\left\{\mathbf{j}^{+}\left(\zeta_{1}\right) \mathbf{j}^{-}\left(\zeta_{2}\right)\left(q^{\alpha H(0)}\right)\right\}=\frac{1}{\mathcal{N}\left(\zeta_{1}\right) \mathcal{N}\left(\zeta_{2}\right)}\left|\begin{array}{cc}
\omega\left(\zeta_{1} q^{1 / 2}, \zeta_{2} q^{1 / 2}\right) & \omega\left(\zeta_{1} q^{1 / 2}, \zeta_{2} q^{-1 / 2}\right) \\
\omega\left(\zeta_{1} q^{-1 / 2}, \zeta_{2} q^{1 / 2}\right) & \omega\left(\zeta_{1} q^{-1 / 2}, \zeta_{2} q^{-1 / 2}\right)
\end{array}\right| .
$$

As has been explained $\mathbf{j}^{+}\left(\zeta_{1}\right) \mathbf{j}^{-}\left(\zeta_{2}\right)\left(q^{\alpha H(0)}\right)$ does not define a two-parametric family of quasi-local operators. It has to be normal ordered. The quasi-local operators are created by

$$
\mathbf{j}^{+}\left(\zeta_{1}\right) \mathbf{j}^{-}\left(\zeta_{2}\right)-\mathbf{j}^{0}\left(\zeta_{2}\right) f\left(\zeta_{1}, \zeta_{2}\right)-f\left(\zeta_{1}, \zeta_{2}\right) f\left(\zeta_{2}, \zeta_{1}\right)
$$

and the value of $Z_{\mathbf{n}}^{\kappa}$ on $\mathbf{j}^{0}(\zeta)\left(q^{\alpha H(0)}\right)$ is given by

$$
Z_{\mathbf{n}}^{\kappa}\left\{\mathbf{j}^{0}(\zeta)\left(q^{\alpha H(0)}\right)\right\}=\frac{1}{\mathcal{N}(\zeta)}\left[\omega\left(\zeta q^{1 / 2}, \zeta q^{-1 / 2}\right)-\omega\left(\zeta q^{-1 / 2}, \zeta q^{1 / 2}\right)\right]_{\mathrm{reg}}
$$

following the regularisation in the definition (3.17).

In principle this kind of formulae allows us to evaluate $Z_{\mathbf{n}}^{\kappa}$ on any quasi-local operator. However, we do not think that for the operators of small length this way of doing adds much to the direct fusion computation of [21]. We rather consider the result of this paper as an existence theorem of a good basis in the space of quasi-local operators. We hope to be able to use this basis for considering the scaling limit which includes the super-symmetric 
CFT and its massive deformation, super sine-Gordon model. From that point of view the present paper should be considered as a preparation for this future investigation.

\section{Appendix A. Review of the Spin 1/2 CASE}

In this appendix we give a summary of the definition and the properties of the operators $g_{c}^{\epsilon}(\zeta)$ used in the main text. In particular, we give a proof of the dressed Russian doll formula.

A.1. Operators $g_{c}^{\epsilon}(\zeta)$. Fix an interval $[1, n] \subset \mathbb{Z}$. For $j \in[1, n]$, denote by $V_{j}\left(\xi_{j}\right)$ the two-dimensional representation of $U_{q}\left(\widehat{\mathfrak{s l}}_{2}\right)$ with evaluation parameter $\xi_{j}$, and set

$$
V_{[1, n]}=V_{1}\left(\xi_{1}\right) \otimes \cdots \otimes V_{n}\left(\xi_{n}\right) .
$$

We shall deal with several linear operators on $\operatorname{End}\left(V_{[1, n]}\right)$.

Let us start from an adjoint action by monodromy matrices. Let $W_{*}(\zeta)$ be an 'auxiliary space' (i.e., a representation of the Borel subalgebra of $\left.U_{q}\left(\widehat{\mathfrak{s l}}_{2}\right)\right)$, and let $L_{*, j}\left(\zeta / \xi_{j}\right)$ be the image of the universal $R$ matrix on $W_{*}(\zeta) \otimes V_{j}(\xi)$. For $X \in \operatorname{End}\left(V_{[1, n]}\right)$, we set

$$
\begin{aligned}
& \mathbb{T}_{*,[1, n]}(\zeta)(X)=T_{*,[1, n]}(\zeta) \cdot X \cdot T_{*,[1, n]}(\zeta)^{-1} \\
& T_{*,[1, n]}(\zeta)=L_{*, n}\left(\zeta / \xi_{n}\right) \cdots L_{*, 1}\left(\zeta / \xi_{1}\right)
\end{aligned}
$$

As for the auxiliary space $W_{*}(\zeta)$, various choices are possible. The ones relevant to us are the two dimensional module $W_{*}(\zeta)=V_{a}(\zeta)$, and the tensor product module $W_{*}(\zeta)=$ $V_{a}(\zeta) \otimes V_{A}(\zeta)$ where $V_{A}(\zeta)$ stands for the representation of the Borel subalgebra by $q$ oscillators

$$
q^{-1} \mathbf{a a}^{*}-q \mathbf{a}^{*} \mathbf{a}=q^{-1}-q, \quad q^{D} \mathbf{a}^{*} q^{-D}=q \mathbf{a}^{*}, \quad q^{D} \mathbf{a} q^{-D}=q^{-1} \mathbf{a} .
$$

For the formulas of the corresponding monodromy matrices $\mathbb{T}_{a,[1, n]}(\zeta)$ and $\mathbb{T}_{\{a, A\},[1, n]}(\zeta)$, the reader is referred to [9], eq.(2.4) and eq.(2.19).

Next we introduce operators $\mathbf{k}(\zeta), \boldsymbol{\ell}(\zeta), \mathbf{f}(\zeta)$ on $\operatorname{End}\left(V_{[1, n]}\right)$ by certain traces involving monodromy matrices. Their definition depends on the spin of the operand $X \in$ $\operatorname{End}\left(V_{[1, n]}\right)$. Here we say that $X$ has spin $s \in \mathbb{Z}$, and write as $s(X)=s$, if $\left[\frac{1}{2} H_{[1, n]}, X\right]=$ $s X$ holds with $H_{[1, n]}=\sum_{j=1}^{n} \sigma_{j}^{3}$.

For $X \in \operatorname{End}\left(V_{[1, n]}\right)$ with $s(X)=s$, define

$$
\begin{aligned}
& \mathbf{k}(\zeta)(X)=\zeta^{\alpha-2 s-1} \operatorname{Tr}_{a, A}\left\{\sigma_{a}^{+} \mathbb{T}_{\{a, A\},[1, n]}(\zeta) q^{(\alpha-s-1)\left(2 D_{A}+\sigma_{a}^{3}\right)}\left(q^{\left.-H_{[1, n]} X\right)}\right\}\right. \\
& \boldsymbol{\ell}(\zeta)(X)=\zeta^{\alpha-2 s-1} \operatorname{Tr}_{a, A}\left\{\left(\sigma_{a}^{3}-\mathbf{a}_{A} \sigma_{a}^{+}\right) \mathbb{T}_{\{a, A\},[1, n]}(\zeta) q^{(\alpha-s-1)\left(2 D_{A}+\sigma_{a}^{3}\right)}\left(q^{\left.-H_{[1, n]} X\right)}\right\}\right. \\
& \mathbf{f}(\zeta)(X)=\Delta_{\zeta}^{-1} \mathbf{k}(\zeta)(X)
\end{aligned}
$$

In the last line the symbol $\Delta_{\zeta}^{-1}$ is defined by the integral

$$
\Delta_{\zeta}^{-1} \mathbf{k}(\zeta)(X)=\int_{\Gamma} \Delta_{\zeta}^{-1} \psi(\zeta / \xi, \alpha) \cdot \mathbf{k}(\xi)(X) \frac{d \xi^{2}}{2 \pi i \xi^{2}}
$$


where the contour $\Gamma$ is such that the poles of $\Delta_{\zeta}^{-1} \psi(\zeta / \xi, \alpha)$ are outside and the poles of $\mathbf{k}(\xi)(X)$ are inside. The function $\xi^{-\alpha} \mathbf{k}(\xi)(X)$ is rational in $\xi^{2}$, behaves as $O\left(\xi^{-2}\right)$ at $\xi^{2}=\infty$, and has poles only at $q^{2} \xi_{j}^{2}, \xi_{j}^{2}, q^{-2} \xi_{j}^{2}(j \in[1, n])$ and at 0 when $s>0$.

Finally, for $\epsilon=0, \overline{0},+,-$ we define the linear maps

$$
g_{c}^{\epsilon}(\zeta): \operatorname{End}\left(V_{[1, n]}\right) \longrightarrow \operatorname{End}\left(V_{[1, n]} \otimes V_{c}(\zeta)\right)
$$

as follows. Assuming $s(X)=s$, we set

$$
\begin{aligned}
g_{c}^{0}(\zeta)(X) & =\mathbb{T}_{c,[1, n]}(\zeta)\left(X \cdot q^{(\alpha-s) \sigma_{c}^{3}}\right), \\
g_{c}^{\overline{0}}(\zeta)(X) & =X \otimes \mathrm{id}, \\
g_{c}^{+}(\zeta)(X) & =\frac{1}{2} \mathbf{f}(q \zeta)(X)+\frac{1}{2} \mathbf{f}\left(q^{-1} \zeta\right)(X)-g_{c}^{0}(\zeta)(\mathbf{f}(\zeta)(X)) \\
& -\frac{1}{2} \sigma_{c}^{3} \cdot \mathbf{k}(\zeta)(X)+\sigma_{c}^{+} \cdot \ell(\zeta)(X), \\
g_{c}^{-}(\zeta)(X) & =-q^{-\alpha+2 s-2}\left(1-q^{2(\alpha-2 s+1)}\right) \times\left.\left\{\left(\mathbb{J} \circ g_{c}^{+}(\zeta) \circ \mathbb{J}\right)(X)\right\}\right|_{\alpha \rightarrow-\alpha+2 s} .
\end{aligned}
$$

When necessary, we exhibit explicitly the dependence on the evaluation parameters and write (A.4) as

$$
g_{c,[1, n]}^{\epsilon}\left(\zeta \mid \xi_{1}, \cdots, \xi_{n}\right) .
$$

In this notation, for example, the composition

$$
\operatorname{End}\left(V_{[1, n]}\right) \longrightarrow \operatorname{End}\left(V_{[1, n]} \otimes V_{c_{1}}\left(\zeta_{1}\right)\right) \longrightarrow \operatorname{End}\left(V_{[1, n]} \otimes V_{c_{1}}\left(\zeta_{1}\right) \otimes V_{c_{2}}\left(\zeta_{2}\right)\right)
$$

is given by

$$
g_{c_{2},[1, n] \cup c_{1}}^{\epsilon_{2}}\left(\zeta_{2} \mid \xi_{1}, \cdots, \xi_{n}, \zeta_{1}\right) \circ g_{c_{1},[1, n]}^{\epsilon_{1}}\left(\zeta_{1} \mid \xi_{1}, \cdots, \xi_{n}\right)
$$

To unburden the notation we often abbreviate (A.9) to $g_{c_{2}}^{\epsilon_{2}}\left(\zeta_{2}\right) g_{c_{1}}^{\epsilon_{1}}\left(\zeta_{1}\right)$, keeping in mind that the space $V_{c_{1}}\left(\zeta_{1}\right)$ is 'added' first and $V_{c_{2}}\left(\zeta_{2}\right)$ the next.

Example 1. In the simplest case when $[1, n]=\emptyset$, the action of $g_{c}^{\epsilon}(\zeta)$ on the unit operator $I$ reads

$$
\begin{aligned}
& g_{c}^{0}(\zeta)(I)=q^{\alpha \sigma_{c}^{3}}, \\
& g_{c}^{+}(\zeta)(I)=-\left(q^{-1} \zeta\right)^{\alpha-1} \sigma_{c}^{+}, \quad g_{c}^{-}(\zeta)(I)=q^{-1}\left(1-q^{2(\alpha+1)}\right) \zeta^{-\alpha-1} \sigma_{c}^{-} .
\end{aligned}
$$

Here and after,

$$
\tau^{+}=\left(\begin{array}{ll}
1 & 0 \\
0 & 0
\end{array}\right), \quad \tau^{-}=\left(\begin{array}{ll}
0 & 0 \\
0 & 1
\end{array}\right), \quad \sigma^{+}=\left(\begin{array}{ll}
0 & 1 \\
0 & 0
\end{array}\right), \quad \sigma^{-}=\left(\begin{array}{ll}
0 & 0 \\
1 & 0
\end{array}\right) .
$$


Example 2. Set $\zeta=\zeta_{2} / \zeta_{1}$. We have

$$
\begin{aligned}
& g_{c_{2}}^{+}\left(\zeta_{2}\right) g_{c_{1}}^{-}\left(\zeta_{1}\right)(I)=\Delta_{\zeta}^{-1} \psi(\zeta, \alpha)\left(I+q^{\alpha\left(\sigma_{c_{1}}^{3}+\sigma_{c_{2}}^{3}\right)}\right) \\
& -\frac{1}{2}\left(\Delta_{\zeta}^{-1} \psi(q \zeta, \alpha)+\Delta_{\zeta}^{-1} \psi\left(q^{-1} \zeta, \alpha\right)\right)\left(q^{\alpha \sigma_{c_{1}}^{3}}+\mathbb{R}_{c_{2}, c_{1}}(\zeta)\left(q^{\alpha \sigma_{c_{2}}^{3}}\right)\right) \\
& -\frac{1}{2} \frac{q^{\alpha}+q^{-\alpha}}{q^{\alpha}-q^{-\alpha}} \psi(\zeta, \alpha)\left(q^{\alpha \sigma_{c_{1}}^{3}}-\mathbb{R}_{c_{2}, c_{1}}(\zeta)\left(q^{\alpha \sigma_{c_{2}}^{3}}\right)\right) \\
& +\psi(q \zeta, \alpha) \tau_{c_{1}}^{+} \tau_{c_{2}}^{-}-\psi\left(q^{-1} \zeta, \alpha\right) \tau_{c_{1}}^{-} \tau_{c_{2}}^{+}-\left(\frac{(q \zeta)^{\alpha}}{q \zeta-q^{-1} \zeta^{-1}}-\frac{\left(q^{-1} \zeta\right)^{\alpha}}{q^{-1} \zeta-q \zeta^{-1}}\right) \sigma_{c_{1}}^{-} \sigma_{c_{2}}^{+}, \\
& g_{c_{2}}^{-}\left(\zeta_{2}\right) g_{c_{1}}^{+}\left(\zeta_{1}\right)(I)=-\left.\left\{\mathbb{J}\left(g_{c_{2}}^{+}\left(\zeta_{2}\right) g_{c_{1}}^{-}\left(\zeta_{1}\right)(I)\right)\right\}\right|_{\alpha \rightarrow-\alpha} .
\end{aligned}
$$

A.2. Operators $g_{\text {rat }, c}^{\epsilon}(\zeta)$. Our convention and notation used here are slightly different from those of [9], [10]. Let us explain the precise relation between the two.

For that matter, we need the annihilation operators

$$
x^{ \pm}(\zeta), \bar{x}^{+}(\zeta): \text { End } V_{[1, n]} \longrightarrow \text { End } V_{[1, n]} .
$$

Define for $X \in$ End $V_{[1, n]}$ with $s(X)=s$

$$
\begin{aligned}
& x^{+}(\zeta)(X)=\int_{\Gamma_{0}} \psi(\zeta / \xi, \alpha) \cdot\left(\mathbf{k}(q \xi)+\mathbf{k}\left(q^{-1} \xi\right)\right)(X) \frac{d \xi^{2}}{2 \pi i \xi^{2}}, \\
& \bar{x}^{+}(\zeta)(X)=\int_{\Gamma_{0}} \psi(\zeta / \xi, \alpha) \cdot \mathbf{k}(\xi)(X) \frac{d \xi^{2}}{2 \pi i \xi^{2}} \\
& x^{-}(\zeta)(X)=q^{-\alpha+2 s-2}\left(1-q^{2(\alpha-2 s+1)}\right) \times\left.\left\{\mathbb{J} \circ x^{+}(\zeta) \circ \mathbb{J}(X)\right\}\right|_{\alpha \rightarrow 2 s-\alpha} .
\end{aligned}
$$

The contour $\Gamma_{0}$ encircles $\xi^{2}=\xi_{j}^{2}$ for all $j$.

Consider the operator

$$
\mathbf{f}_{\mathrm{rat}}(\zeta)(X)=\int_{\Gamma} \Delta^{-1} \psi(\zeta / \xi, \alpha) \cdot\left(\mathbf{k}(\xi)-\bar{x}^{+}(\xi)-\frac{1}{2}\left(x^{+}(q \xi)+x^{+}\left(q^{-1} \xi\right)\right)\right)(X) \frac{d \xi^{2}}{2 \pi i \xi^{2}} .
$$

Define further $g_{\mathrm{rat}, c}^{ \pm}(\zeta)$ by replacing $\mathbf{f}(\zeta)$ by $\mathbf{f}_{\mathrm{rat}}(\zeta)$ in the definition (A.7), (A.8). Then the coefficients of $g_{\text {rat }, c}^{ \pm}(\zeta)$ are rational functions of $\zeta^{2}$ (up to an overall power).

Let us write $\mathbf{b}_{I I,[1, n]}^{*}(\zeta, \alpha)$, etc. for the operators defined in [9] in the inhomogeneous case. Then, for $X \in$ End $V_{[1, n]}$ with $s(X)=s$, we have

$$
\begin{aligned}
& g_{\mathrm{rat}, c}^{+}(\zeta)(X)=\frac{1}{2} \mathbf{b}_{I I,[1, n] \cup c}^{*}(\zeta, \alpha-s-1)(X), \\
& g_{\mathrm{rat}, c}^{-}(\zeta)(X)=\frac{1}{2} \mathbf{c}_{I I,[1, n] \cup c}^{*}(\zeta, \alpha-s+1)(X), \\
& g_{c}^{0}(\zeta)(X)=\frac{1}{2} \mathbf{t}_{I I,[1, n] \cup c}^{*}(\zeta, \alpha-s)(X), \\
& x^{+}(\zeta)(X)=2 \mathbf{c}_{I I,[1, n]}(\zeta, \alpha-s-1)(X), \\
& x^{-}(\zeta)(X)=2 \mathbf{b}_{I I,[1, n]}(\zeta, \alpha-s+1)(X) .
\end{aligned}
$$


In the right hand side, the interval $[1, n] \cup c$ signifies $V_{1}\left(\xi_{1}\right) \otimes \cdots \otimes V_{n}\left(\xi_{n}\right) \otimes V_{c}(\zeta)$. The factor 2 appears because we use the normalised trace in this paper.

A.3. Dual rqKZ equation. In addition to $g_{c}^{0}(\zeta), g_{c}^{ \pm}(\zeta)$, we use the tautological operator $g_{c}^{\overline{0}}(\zeta)$ defined in (3.13). Acting with (A.5) (A.8) on the unit operator $I$ we obtain a set of $4^{n}$ operators

$$
g^{\epsilon_{n}, \cdots, \epsilon_{1}}\left(\xi_{n}, \cdots, \xi_{1}\right)=g_{n}^{\epsilon_{n}}\left(\xi_{n}\right) \cdots g_{1}^{\epsilon_{1}}\left(\xi_{1}\right)(I) \quad\left(\epsilon_{1}, \cdots, \epsilon_{n} \in\{0, \overline{0},+,-\}\right) .
$$

For generic $\xi_{1}, \cdots, \xi_{n}$ this gives a basis of End $V_{[1, n]}$ (see [9], Lemma 5.1).

The basis (A.13) has a distinguished feature that it satisfies the dual rqKZ equation

$$
\begin{aligned}
& g^{\epsilon_{n}, \cdots, \epsilon_{j}, \cdots, \epsilon_{1}}\left(\xi_{n}, \cdots, \xi_{j}, \cdots, \xi_{1}\right) \\
& =(-1)^{\epsilon_{j}} \mathbb{A}_{j,[1, n]}\left(\xi_{1}, \cdots, \xi_{n}\right) \circ g^{\epsilon_{n}, \cdots, \bar{\epsilon}_{j}, \cdots, \epsilon_{1}}\left(\xi_{n}, \cdots, \xi_{j} q^{-1}, \cdots, \xi_{1}\right),
\end{aligned}
$$

where we set $\bar{\epsilon}=\epsilon$ for $\epsilon= \pm$. Operators $\mathbb{A}_{j,[1, n]}$ are defined by (4.2).

The dual rqKZ equation (A.14) is a consequence of two identities, which for historical reasons we call the first and the second difference equations.

The first difference equation states that

$$
\begin{aligned}
& g_{n,[1, n-1]}^{\epsilon}\left(\xi_{n} \mid \xi_{1}, \cdots, \xi_{n-1}\right)\left(X_{[1, n-1]}\right) \\
& =(-)^{\epsilon} \mathbb{A}_{n,[1, n]}\left(\xi_{1}, \cdots, \xi_{n}\right)\left(g_{n,[1, n-1]}^{\bar{\epsilon}}\left(q^{-1} \xi_{n} \mid \xi_{1}, \cdots, \xi_{n-1}\right)\left(X_{[1, n-1]}\right)\right)
\end{aligned}
$$

for $X_{[1, n-1]}$ which acts as identity on the $n$-th component. For $\epsilon=0, \overline{0}$, this is a restatement of (4.4), (4.5). The essential case $\epsilon=+$ has been proved in [10], Lemma B.2.

The second difference equation

$$
\begin{aligned}
& g_{n,[1, n-1]}^{\epsilon}\left(\xi_{n} \mid \xi_{1}, \cdots, \xi_{n-1}\right) \circ \mathbb{A}_{n-1,[1, n-1]}\left(\xi_{1}, \cdots, \xi_{n-1}\right) \\
& =\mathbb{A}_{n-1,[1, n]}\left(\xi_{1}, \cdots, \xi_{n}\right) \circ g_{n,[1, n-1]}^{\epsilon}\left(\xi_{n} \mid \xi_{1}, \cdots, q^{-1} \xi_{n-1}\right)
\end{aligned}
$$

was not used in [10], but can be deduced in a similar (and simpler) way as for (A.15).

A.4. Inductive limit. Being constructed through the monodromy matrices, $g_{c}^{\epsilon}(\zeta)$ have the $R$ matrix symmetry. Namely we have

$$
\begin{aligned}
& g_{c,[1, n]}^{\epsilon}\left(\zeta_{1} \mid \xi_{1}, \cdots, \xi_{j}, \xi_{j+1}, \cdots, \xi_{n}\right) \mathbb{R}_{j+1, j}\left(\xi_{j+1} / \xi_{j}\right) \\
& =\mathbb{R}_{j+1, j}\left(\xi_{j+1} / \xi_{j}\right) g_{c,[1, j-1] \cup\{j+1\} \cup\{j\} \cup[j+2, n]}^{\epsilon}\left(\zeta_{1} \mid \xi_{1}, \cdots, \xi_{j+1}, \xi_{j}, \cdots, \xi_{n}\right) .
\end{aligned}
$$

Operators $g_{c}^{\epsilon}(\zeta)$ enjoy also the following right reduction property: If $n<N$ and $X_{[1, n]} \in$ End $V_{[1, n]}$, then

$$
g_{c,[1, N]}^{\epsilon}\left(\zeta \mid \xi_{1}, \cdots, \xi_{N}\right)\left(X_{[1, n]}\right)=\mathbb{T}_{c,[n+1, N]}\left(\zeta \mid \xi_{n+1}, \cdots, \xi_{N}\right) g_{c,[1, n]}^{\epsilon}\left(\zeta \mid \xi_{1}, \cdots, \xi_{n}\right)\left(X_{[1, n]}\right)
$$

For $\epsilon=0$ this is immediate. The non-trivial case $\epsilon=+$ follows from Lemma 3.7 in [9]. In [9] we considered $g_{\mathrm{rat}, c}^{\epsilon}(\zeta)$, but this equation is equally valid for $g_{c}^{\epsilon}(\zeta)$ since they differ only by annihilation operators. 
From (A.18) we can deduce the reduction property for the composition (A.9):

$$
\begin{aligned}
g_{c_{2},[1, N] \cup c_{1}}^{\epsilon_{2}}\left(\zeta_{2} \mid \xi_{1}, \cdots, \xi_{N}, \zeta_{1}\right) g_{c_{1},[1, N]}^{\epsilon_{1}}\left(\zeta_{1} \mid \xi_{1}, \cdots, \xi_{N}\right)\left(X_{[1, n]}\right) \\
=g_{c_{2},[1, N] \cup c_{1}}^{\epsilon_{2}}\left(\zeta_{2} \mid \xi_{1}, \cdots, \xi_{N}, \zeta_{1}\right) \mathbb{T}_{c_{1},[n+1, N]}\left(\zeta_{1} \mid \xi_{n+1}, \cdots, \xi_{N}\right) g_{c_{1},[1, n]}^{\epsilon_{1}}\left(\zeta_{1} \mid \xi_{1}, \cdots, \xi_{n}\right)\left(X_{[1, n]}\right) \\
=\mathbb{T}_{c_{1},[n+1, N]}\left(\zeta_{1} \mid \xi_{n+1}, \cdots, \xi_{N}\right) g_{c_{2},[1, n] \cup c_{1} \cup[n+1, N]}^{\epsilon_{2}}\left(\zeta_{2} \mid \xi_{1}, \cdots, \xi_{n}, \zeta_{1}, \xi_{n+1}, \cdots, \xi_{N}\right) \\
\quad \times g_{c_{1},[1, n]}^{\epsilon_{1}}\left(\zeta_{1} \mid \xi_{1}, \cdots, \xi_{n}\right)\left(X_{[1, n]}\right) \\
=\mathbb{T}_{c_{1},[n+1, N]}\left(\zeta_{1} \mid \xi_{n+1}, \cdots, \xi_{N}\right) \mathbb{T}_{c_{2},[n+1, N]}\left(\zeta_{2} \mid \xi_{n+1}, \cdots, \xi_{N}\right) \\
\quad \times g_{c_{2},[1, n] \cup c_{1}}^{\epsilon_{2}}\left(\zeta_{2} \mid \xi_{1}, \cdots, \xi_{n}, \zeta_{1}\right) g_{c_{1},[1, n]}^{\epsilon_{1}}\left(\zeta_{1} \mid \xi_{1}, \cdots, \xi_{n}\right)\left(X_{[1, n]}\right) .
\end{aligned}
$$

In the third line we used the $R$ matrix symmetry (A.17).

Now let us fix $\left\{\xi_{j}\right\}_{j=1}^{\infty}$ in such a way that $\xi_{j}=1$ for $j \gg 1$. The right hand side of (A.18) can be rewritten as

$$
\check{\mathbb{R}}_{c, N}\left(\zeta / \xi_{N}\right) \cdots \check{\mathbb{R}}_{n+2, n+1}\left(\zeta / \xi_{n+1}\right) g_{n+1,[1, n]}^{\epsilon}\left(\zeta \mid \xi_{1}, \cdots, \xi_{n}\right)\left(X_{[1, n]}\right) .
$$

Therefore, by the same argument as in Section 2, we see that the inductive limit

$$
g^{\epsilon}(\zeta)\left(X_{[1, n]}\right):=\lim _{N \rightarrow \infty} g_{c,[1, N]}^{\epsilon}\left(\zeta \mid \xi_{1}, \cdots, \xi_{n}\right)\left(X_{[1, n]}\right)
$$

is well defined as a formal series in $\zeta^{2}-1$. For any given $p$

$$
\begin{aligned}
& g^{\epsilon}(\zeta)\left(X_{[1, n]}\right) \\
& \quad \equiv \operatorname{Tr}_{c}\left\{\mathbb{T}_{c,[n+1, N]}\left(\zeta \mid \xi_{n+1}, \cdots, \xi_{N}\right) g_{c,[1, n]}^{\epsilon}\left(\zeta \mid \xi_{1}, \cdots, \xi_{n}\right)\left(X_{[1, n]}\right)\right\} \quad \bmod \left(\zeta^{2}-1\right)^{p},
\end{aligned}
$$

holds for $N$ large enough.

Letting $N \rightarrow \infty$ in (A.19), we conclude that

$$
\begin{aligned}
& g^{\epsilon_{2}}\left(\zeta_{2}\right) g^{\epsilon_{1}}\left(\zeta_{1}\right)\left(X_{[1, n]}\right) \\
& \quad=\operatorname{Tr}_{c_{1}, c_{2}}\left\{\mathbb{T}_{c_{1},[n+1, \infty)}\left(\zeta_{1}\right) \mathbb{T}_{c_{2},[n+1, \infty)}\left(\zeta_{2}\right) g_{c_{2}}^{\epsilon_{2}}\left(\zeta_{2}\right) g_{c_{1}}^{\epsilon_{1}}\left(\zeta_{1}\right)\left(X_{[1, n]}\right)\right\} .
\end{aligned}
$$

Obviously we have in general

$$
\begin{aligned}
& g^{\epsilon_{k}}\left(\zeta_{k}\right) \cdots g^{\epsilon_{1}}\left(\zeta_{1}\right)\left(X_{[1, n]}\right) \\
& \quad=\operatorname{Tr}_{c_{1}, \cdots, c_{k}}\left\{\mathbb{T}_{c_{1},[n+1, \infty)}\left(\zeta_{1}\right) \cdots \mathbb{T}_{c_{k},[n+1, \infty)}\left(\zeta_{k}\right) g_{c_{k}}^{\epsilon_{k}}\left(\zeta_{k}\right) \cdots g_{c_{1}}^{\epsilon_{1}}\left(\zeta_{1}\right)\left(X_{[1, n]}\right)\right\} .
\end{aligned}
$$

Let us give a remark about the commutation relations. Rewriting the right hand side of (A.21) as

$$
\operatorname{Tr}_{c_{1}, c_{2}}\left\{\mathbb{T}_{c_{2},[n+1, \infty)}\left(\zeta_{2}\right) \mathbb{T}_{c_{1},[n+1, \infty)}\left(\zeta_{1}\right) \mathbb{R}_{c_{1}, c_{2}}\left(\zeta_{1} / \zeta_{2}\right) g_{c_{2}}^{\epsilon_{2}}\left(\zeta_{2}\right) g_{c_{1}}^{\epsilon_{1}}\left(\zeta_{1}\right)\left(X_{[1, n]}\right)\right\}
$$

and comparing it with $g^{\epsilon_{1}}\left(\zeta_{1}\right) g^{\epsilon_{2}}\left(\zeta_{2}\right)\left(X_{[1, n]}\right)$, we see that

$$
g^{\epsilon_{1}}\left(\zeta_{1}\right) g^{\epsilon_{2}}\left(\zeta_{2}\right)=(-)^{\epsilon_{1} \epsilon_{2}} g^{\epsilon_{2}}\left(\zeta_{2}\right) g^{\epsilon_{1}}\left(\zeta_{1}\right)
$$

is equivalent to

$$
g_{c_{1}}^{\epsilon_{1}}\left(\zeta_{1}\right) g_{c_{2}}^{\epsilon_{2}}\left(\zeta_{2}\right)=(-)^{\epsilon_{1} \epsilon_{2}} \mathbb{R}_{c_{1}, c_{2}}\left(\zeta_{1} / \zeta_{2}\right) g_{c_{2}}^{\epsilon_{2}}\left(\zeta_{2}\right) g_{c_{1}}^{\epsilon_{1}}\left(\zeta_{1}\right)
$$

In the main text, we extend (A.20) further to define operators acting on the space $\mathcal{W}^{(\alpha)}$ of quasi-local operators,

$$
x^{\epsilon *}(\zeta)\left(q^{(\alpha-s+\epsilon) H(0)} \mathcal{O}\right)=q^{(\alpha-s) H(0)} g^{\epsilon}(\zeta)\left(\mathcal{O}_{[1, n]}\right),
$$


where $\mathcal{O}$ is supported on $[1, n]$. The relation (A.23) is nothing but the (anti-)commutativity between $b^{*}(\zeta)=x^{+*}(\zeta), c^{*}(\zeta)=x^{-*}(\zeta)$ and $t^{*}(\zeta)=x^{0 *}(\zeta)$. In this latter form the commutation relations have been proved in [23] by an indirect argument based on the results of [10].

A.5. Expectation values. The main result of [10] states that the expectation values of quasi-local operators in the fermionic basis are expressed as determinants of the simplest one:

$$
\omega(\zeta, \xi)=Z_{\mathbf{n}}^{\kappa}\left\{b^{*}(\zeta) c^{*}(\xi)\left(q^{\alpha H(0)}\right)\right\}
$$

The precise relation between this function and the one in [10] is as follows.

We assume that in the Matsubara direction there are $\mathbf{n}$ number of rows corresponding to representations of dimension $d_{\mathbf{m}}$ and evaluation parameters $\boldsymbol{\tau}_{\mathbf{m}}$, and set

$$
a(\zeta)=\prod_{\mathbf{m}=1}^{\mathbf{n}}\left(q^{d_{\mathbf{m}}} \zeta^{2} / \boldsymbol{\tau}_{\mathbf{m}}^{2}-1\right), \quad d(\zeta)=\prod_{\mathbf{m}=1}^{\mathbf{n}}\left(q^{2-d_{\mathbf{m}}} \zeta^{2} / \boldsymbol{\tau}_{\mathbf{m}}^{2}-1\right) .
$$

Denote by $\omega_{I I I}(\zeta, \xi)$ the function defined in [10], eq.(7.2). Then we have

$$
\omega(\zeta, \xi)=\frac{1}{4} \omega_{I I I}(\zeta, \xi)+\frac{1}{4} \bar{D}_{\zeta} \bar{D}_{\xi} \Delta_{\zeta}^{-1} \psi(\zeta / \xi, \alpha)
$$

where

$$
\begin{aligned}
& \bar{D}_{\zeta} f(\zeta)=f(\zeta q)+f\left(\zeta q^{-1}\right)-2 \rho(\zeta) f(\zeta), \\
& \rho(\zeta)=\frac{T(\zeta, \kappa+\alpha)}{T(\zeta, \kappa)}
\end{aligned}
$$

The function $\omega(\zeta, \xi)$ has singularities at $\zeta=\xi q^{ \pm 1}$, with the singular part given by

$$
\begin{aligned}
& \frac{1}{4} \omega_{I I I, \text { symm }}(\zeta, \xi)+\frac{1}{4} \bar{D}_{\zeta} \bar{D}_{\xi} \Delta_{\zeta}^{-1} \psi(\zeta / \xi, \alpha) \\
& =(1+\rho(\zeta) \rho(\xi)) \Delta_{\zeta}^{-1} \psi(\zeta / \xi, \alpha)-\rho(\xi) \Delta_{\zeta}^{-1} \psi(q \zeta / \xi, \alpha)-\rho(\zeta) \Delta_{\zeta}^{-1} \psi\left(q^{-1} \zeta / \xi, \alpha\right) \\
& +\frac{1}{4} \frac{a(\xi) d(\zeta)}{T(\xi, \kappa) T(\zeta, \kappa)} \psi(q \zeta / \xi, \alpha)-\frac{1}{4} \frac{a(\zeta) d(\xi)}{T(\zeta, \kappa) T(\xi, \kappa)} \psi\left(q^{-1} \zeta / \xi, \alpha\right) .
\end{aligned}
$$

\section{Appendix B. Admissibility of $g_{c_{1}, c_{2}}^{(2,2)}(\zeta)$}

We show here the admissibility of $g_{c_{1}, c_{2}}^{(2,2)}(\zeta)$. Consider the combination

$$
G\left(\zeta_{2}, \zeta_{1}\right)=g_{c_{2}}^{+}\left(\zeta_{2}\right) g_{c_{1}}^{-}\left(\zeta_{1}\right)+g_{c_{2}}^{-}\left(\zeta_{2}\right) g_{c_{1}}^{+}\left(\zeta_{1}\right)
$$

The possible poles of $G\left(\zeta_{2}, \zeta_{1}\right)$ in $\zeta_{2} / \zeta_{1}$ are at the shifted diagonal $\zeta_{2} / \zeta_{1}=q^{m}$. Among them, $m=0$ is known to be absent [9]. In addition, the commutation relation implies

$$
G(\zeta, \zeta)=0 \text {. }
$$


On the other hand, the dual rqKZ equation reads

$$
\begin{aligned}
G\left(\zeta_{2}, \zeta_{1}\right) & =-R_{c_{2}, c_{1}}\left(\zeta_{2} / \zeta_{1}\right) q^{\alpha H_{c_{2}}} \theta_{c_{2}}\left(G\left(\zeta_{2} q^{-1}, \zeta_{1}\right) \theta_{c_{2}}\left(R_{c_{2}, c_{1}}\left(\zeta_{2} / \zeta_{1}\right)^{-1}\right)\right) \\
& =-q^{\alpha H_{c_{1}}} \theta_{c_{1}}\left(\theta_{c_{1}}\left(R_{c_{1}, c_{2}}\left(\zeta_{1} / \zeta_{2}\right)^{-1}\right) G\left(\zeta_{2}, \zeta_{1} q^{-1}\right)\right) R_{c_{1}, c_{2}}\left(\zeta_{1} / \zeta_{2}\right) .
\end{aligned}
$$

Combining (B.1), (B.2) we find that $G\left(\zeta_{2}, \zeta_{1}\right)$ is in fact regular at $\zeta_{2} / \zeta_{1}=q^{m}$ for all $m \in \mathbb{Z}$. Moreover the first equation of (B.2) implies that $G(q \zeta, \zeta)=\mathcal{P}_{c_{1}, c_{2}}^{+} G(q \zeta, \zeta)$, showing that $G(q \zeta, \zeta)$ is admissible.

\section{Acknowledgements.}

MJ and TM would like to thank Andreas Klümper and Junji Suzuki for discussions. Research of MJ is supported by the Grant-in-Aid for Scientific Research B-23340039. Research of TM is supported by the Grant-in-Aid for Scientific Research B-22340031. Research of FS is supported by DIADEMS program (ANR) contract number BLAN012004.

\section{REFERENCES}

[1] H. Boos and V. Korepin. Quantum spin chains and Riemann zeta functions with odd arguments. J. Phys., A 34:5311-5316, 2001.

[2] H. Boos and V. Korepin. Evaluation of integrals representing correlations in XXX Heisenberg spin chain. MathPhys Odessey 2001,Birkhäuser, pages 65-108, 2001.

[3] M. Jimbo and T. Miwa. Algebraic Analysis of Solvable Lattice Models, volume 85. AMS, 1995.

[4] N. Kitanine, J.-M. Maillet, and V. Terras. Correlation functions of the XXZ Heisenberg spin- $\frac{1}{2}$-chain in a magnetic field. Nucl. Phys. B, 567:554-582, 2000.

[5] H. Boos, V. Korepin, and F. Smirnov. Emptiness formation probability and quantum KnizhnikZamlodchikov equation. Nucl. Phys. B, 658:417-439, 2003.

[6] H. Boos, M. Jimbo, T. Miwa, F. Smirnov, and Y. Takeyama. A recursion formula for the correlation functions of an inhomogeneous XXX model. Algebra and Analysis, 17:115-159, 2005.

[7] H. Boos, M. Jimbo, T. Miwa, F. Smirnov, and Y. Takeyama. Reduced qKZ equation and correlation functions of the XXZ model. Commun. Math. Phys., 261:245-276, 2006.

[8] H. Boos, M. Jimbo, T. Miwa, F. Smirnov, and Y. Takeyama. Hidden Grassmann structure in the XXZ model. Commun. Math. Phys., 272:263-281, 2007.

[9] H. Boos, M. Jimbo, T. Miwa, F. Smirnov, and Y. Takeyama. Hidden Grassmann structure in the XXZ model II : Creation operators. Commun. Math. Phys., 286:875-932, 2009.

[10] M. Jimbo, T. Miwa, and F. Smirnov. Hidden Grassmann structure in the XXZ model III: Introducing Matsubara direction. J. Phys. A:Math.Theor., 42:304018, 2009.

[11] M. Rigol, V. Dunjko, V. Yurovsky, and M. Olshanii. Relaxation in a completely integrable manybody quantum system: An ab initio study of the dynamics of the highly excited states of $1 \mathrm{D}$ lattice hard-core bosons. Phys. Rev. Lett., 98:050405, 2007.

[12] H. Boos, M. Jimbo, T. Miwa, and F. Smirnov. Hidden Grassmann structure in the XXZ model IV: CFT limit. Commun. Math. Phys., 299:825-866, 2010.

[13] M. Jimbo, T. Miwa, and F. Smirnov. On one-point functions of descendants in sine-Gordon model. New Trends in Quantum Integrable Systems: Proceedings of the Infinite Analysis 09, World Scientific Publishing, Singapore, pages 117-137, 2010.

[14] M. Jimbo, T. Miwa, and F. Smirnov. Hidden Grassmann structure in the XXZ model V: sine-Gordon model. Lett. Math. Phys., 96:325-365, 2011.

[15] Al. Zamolodchikov. Two point correlation function in scaling Lee-Yang model. Nucl. Phys., B348:619-641, 1991.

[16] S. Negro and F. Smirnov. Reflection equation and fermionic basis. arXiv:1304.1860 [hep-th], 2013. 
[17] S. Negro and F. Smirnov. On one-point functions for sinh-gordon model at finite temperature. arXiv:1306.1476 [hep-th], 2013.

[18] V. Fateev, D. Fradkin, S. Lukyanov, A. Zamolodchikov, and Al. Zamolodchikov. Expectation values of descendent fields in the sine-Gordon model. Nucl. Phys., B540:587-609, 1999.

[19] A. Zamolodchikov and V. Fateev. A model factorized S-matrix and an integrable spin-1 Heisenberg chain. Sov. J. Nucl. Phys., 32:298-303, 1980.

[20] N. Kitanine. Correlation functions of the higher spin XXX chains. J. Phys. A: Math. Gen., 34:8151, 2001.

[21] A. Klümper, D. Nawrah, and J. Suzuki. Correlation functions of the integrable isotropic spin 1 chain: algebraic expressions for arbitrary temperature. arXiv:1304.5512v1, 2013.

[22] H. Boos, M. Jimbo, T. Miwa, F. Smirnov, and Y. Takeyama. Fermionic basis for space of operators in the XXZ model. SISSA Proceedings of Science, pages Paper 015, 34 pp. (electronic), 2007.

[23] M. Jimbo, T. Miwa, and F. Smirnov. Fermions acting on quasi-local operators in the XXZ model. Symmetries, Integrable Systems and Representations Eds. K.Iohara, S.Morier-Genoud and B. Remy, Springer Proceedings in Mathematics and Statistics, 40:243-261, 2013.

[24] F. Smirnov. Form Factors in Completely Integrable Models of Quantum Field Theory. World Scientific, 1992.

MJ: Department of Mathematics, Rikkyo University, Toshima-ku, Tokyo 171-8501, JAPAN

E-mail address: jimbomm@rikkyo.ac.jp

TM: Department of Mathematics, Graduate School of Science, Kyoto University, КYOTO 606-8502, JAPAN

E-mail address: tmiwa@math.kyoto-u.ac.jp

FS: Sorbonne Université, UPMC Univ Paris 06, CNRS, UMR 7589, LPTHE, F-75005, PARIS, FRANCE

E-mail address: smirnov@lpthe.jussieu.fr 